\title{
ON THE NEAREST SINGULAR MATRIX PENCIL
}

\author{
NICOLA GUGLIELMI*, CHRISTIAN LUBICH ${ }^{\dagger}$, AND VOLKER MEHRMANN ${ }^{\ddagger}$
}

\begin{abstract}
Given a regular matrix pencil $A+\mu E$, we consider the problem of determining the nearest singular matrix pencil with respect to the Frobenius norm. We present new approaches based on the solution of matrix differential equations for determining the nearest singular pencil $A+\Delta A+\mu(E+\Delta E)$, one approach for general singular pencils and another one such that $A+\Delta A$ and $E+\Delta E$ have a common left/right null vector. For the latter case the nearest singular pencil is shown to differ from the original pencil by rank-one matrices $\Delta A$ and $\Delta E$. In both cases we consider also the situation where only $A$ is perturbed. The nearest singular pencil is approached by a two-level iteration, where a gradient flow is driven to a stationary point in the inner iteration and the outer level uses a fast iteration for the distance parameter. This approach extends also to structured matrices $A$ and $E$.
\end{abstract}

Key words. Regular matrix pencil, singular matrix pencil, differential-algebraic equation, lowrank perturbation, matrix differential equation.

AMS subject classifications. 15A18, 65K05

1. Introduction. Let $A, E \in \mathbb{C}^{n, n}$ form a regular matrix pencil $(A, E)$, that is, the linear combination $A+\mu E$ is nonsingular for at least some value $\mu \in \mathbb{C}$. It is a long-standing open problem, see [2], to determine the nearest singular matrix pencil $(A+\Delta A, E+\Delta E)$ (in the sense of $\|(\Delta A, \Delta E)\| \rightarrow \min$ ), i.e., with $\operatorname{det}(A+\Delta A+\mu(E+\Delta E)) \equiv 0$ for all $\mu \in \mathbb{C}$. The norm of this smallest perturbation is called the distance to singularity of the pencil $(A, E)$, and it is of great importance in many applications to have good knowledge about the value of this distance. This holds, in particular, in the analysis of differential-algebraic equation (DAE) models,

$$
F(t, x, \dot{x})=0,
$$

where $\dot{x}$ denotes the time derivative of the state $x$, see e.g. $[1,12,13,17,18,24]$. If DAEs are linearized along a stationary solution, [3], then one obtains a linear DAE

$$
E \dot{x}+A x=f,
$$

with constant coefficients $A, E \in \mathbb{C}^{n \times n}$. If the pencil $(A, E)$ is singular, then the initial value problem of solving (1.2) with a consistent initial value $x(0)=x^{0}$, is not solvable and/or the solution is not unique. Thus, a singular pencil indicates that (1.1) is not well-posed, and the distance to singularity of $(A, E)$ is a robustness measure for the DAE in the neighborhood of a stationary solution.

In many engineering applications, modeling packages such as simscape and modelica [20,23] are employed to automatically generate DAE models of the form (1.1) and it is not clear a priori whether the resulting model is well-posed and adequate for numerical simulation. Since automatically generated DAE models typically contain modeling errors or uncertainties, one usually checks the regularity of the pencil

\footnotetext{
*Dipartimento di Matematica Pura ed Applicata, Università degli Studi di L' Aquila, Via Vetoio - Loc. Coppito, and Gran Sasso Science Institute (GSSI), I-67010 L' Aquila, Italy. Email: guglielm@univaq.it

${ }^{\dagger}$ Mathematisches Institut, Universität Tübingen, Auf der Morgenstelle 10, D-72076 Tübingen, Germany. Email: lubich@na.uni-tuebingen.de

${ }^{\ddagger}$ Institut für Mathematik, MA 4-5, TU Berlin, Str. der 17 Juni 136, D-10623 Berlin, Germany. Email: mehrmann@math.tu-berlin.de
} 
$A+\mu E$ associated with the linearization either in an a priori analysis phase or even at every integration step, since a system with a pencil $A+\mu E$ that is close to a singular pencil within the uncertainty of the data or rounding and discretization errors behaves essentially like a singular system, and so the results of numerical simulations cannot be trusted.

In summary, we discuss the problem of computing the distance to singularity

$$
d(A, E)=\min \{\|(\Delta A, \Delta E)\|:(A+\Delta A, E+\Delta E) \text { is singular }\},
$$

where we consider as metric the Frobenius norm $\|(\Delta A, \Delta E)\|=\|[\Delta A, \Delta E]\|_{F}$.

In many applications, in particular those arising from network analysis, see e.g. [24], or multi-body system simulation, see e.g. [4], the physical application restricts the perturbations of the pencil $A+\mu E$, where data uncertainties arise. Thus, it is also of great interest to study the case that the perturbations are restricted, e.g., by perturbing only one of the matrices $E, A$, by allowing only real perturbations, by allowing only low rank perturbations or by requiring symmetries in the perturbations [21].

An important special case is that the matrix $E$ is not perturbed at all, i.e., $\Delta E \equiv 0$. For example, in certain models from circuit simulation [24], often the matrix $E$ is a matrix with entries $\{0,1,-1\}$, which describes the network topology and therefore is typically not influenced by possible parameter uncertainties. Another important class of applications is that of semi-explicit DAEs $[1,12,13]$, where $E=$ $\operatorname{diag}(1, \ldots, 1,0, \ldots, 0)$. In these examples and many others, it is appropriate to study perturbations only in the matrix $A$, and then we consider the distance

$$
d_{A}(A, E)=\min \{\|\Delta A\|:(A+\Delta A, E) \text { is singular }\} .
$$

We use the convention that $d_{A}(A, E)=\infty$ if $E$ is nonsingular.

The computation of the distances $d(A, E)$ and $d_{A}(A, E)$ is a challenging open problem $[2,16]$ and only in few special cases computational methods have been developed, see $[2,21]$. The reason that this problem is difficult arises from the fact that the nearest singular pencil may be one which has a higher dimensional singular block in its Kronecker canonical form (KCF), see [5], and it is not clear a priori which block structure is the one which is closest.

Calling vectors in the kernel of a matrix null vectors of the matrix, it is seen from the KCF that for singular pencils, $E$ and $A$ may have a common left or right null vector or not. To deal with the first case, we introduce the distance to a pencil with common null vectors

$$
\begin{array}{r}
d^{0}(A, E)=\min \{\|(\Delta A, \Delta E)\|: A+\Delta A, E+\Delta E \\
\text { have a common left or right null vector }\},
\end{array}
$$

and again the analogous distance when only $A$ is perturbed,

$$
\begin{array}{r}
d_{A}^{0}(A, E)=\min \{\|\Delta A\|: A+\Delta A, E \\
\text { have a common left or right null vector }\},
\end{array}
$$

In Section 2 we describe the general methodology of eigenvalue optimization based on solving ordinary differential equations (ODEs) whose trajectories follow descent directions of a suitably chosen minimization functional over perturbations of a given norm, and to optimize this norm in a fast outer iteration; cf. [7]-[10]. Here we extend 
this approach to the problem of computing the distance to singularity. In Section 3 we consider the special situation that the perturbed pencil is required to have a common nullspace but only one of the matrices is perturbed, while the common nullspace problem with perturbations in both matrices is studied in Section 4 .

Notation: For matrices $A=\left[a_{i, j}\right], B \in \mathbb{C}^{n, n}$ we denote by $\Lambda(A)$ the spectrum of $A$, and by $\langle A, B\rangle=\operatorname{trace}\left(A^{H} B\right)$ the inner product corresponding to the Frobenius norm $\|A\|_{F}=\left(\sum_{i, j=1}^{n}\left|a_{i, j}\right|^{2}\right)^{1 / 2}$. Here $A^{H}$ denotes the conjugate transpose of $A$.

2. An ODE-based approach to computing the distance to singularity. To compute the distances $d(A, E), d_{A}(A, E)$ and $d^{0}(A, E), d_{A}^{0}(A, E)$, we use an approach that is based on inner-outer iteration. The inner method uses the numerical integration of the gradient system of a suitable optimization function for perturbations $(\Delta A, \Delta E)$ of a prescribed norm $\varepsilon$, and the outer method is a fast Newton-like iteration for finding the smallest perturbation size $\varepsilon$ that makes the pencil singular. Two-level iterations of a similar type have previously been used in [7]-[10] for other matrix-nearness problems.

In order to deal with eigenvalue optimization, we first recall a classical variational result. The derivative of a simple eigenvalue and an associated eigenvector of a matrix $C(t)$ with respect to variations in a real parameter $t$ of the entries is well-studied, see e.g. [15]. Using the notation $\dot{C}(t):=\frac{d}{d t} C(t)$ to denote the derivative with respect to $t$, we have the following result.

Lemma 2.1. [15, Section II.1.1] Consider a continuously differentiable function $C(t): \mathbb{R} \rightarrow \mathbb{C}^{n, n}$, let $\lambda(t)$ be a simple eigenvalue of $C(t)$ and let $x(t)$ and $y(t)$ be the associated right and left eigenvectors. Then, $y(t)^{H} x(t) \neq 0$ and $\lambda(t)$ is differentiable with

$$
\dot{\lambda}(t)=\frac{y(t)^{H} \dot{C}(t) x(t)}{y(t)^{H} x(t)} .
$$

To check singularity for a matrix pencil $(A, E)$ we use the following characterization of a singular pencil. Let $\mu_{1}, \ldots, \mu_{d} \in \mathbb{C}$ be $d \geq n+1$ distinct numbers, chosen arbitrarily. Then, as a direct consequence of the fundamental theorem of algebra, the pencil $(A+\Delta A, E+\Delta E)$ is singular if and only if

$$
\operatorname{det}\left(A+\Delta A+\mu_{i}(E+\Delta E)\right)=0 \text { for all } i=1, \ldots, d .
$$

Thus, we can recast the problem of computing the distance to singularity as computing a minimum norm pencil $\left(\Delta A_{*}, \Delta E_{*}\right)$ satisfying

$$
\begin{aligned}
\left(\Delta A_{*}, \Delta E_{*}\right)= & \arg \min _{\Delta A, \Delta E \in \mathbb{C}^{n, n}}\|[\Delta A, \Delta E]\| \quad \text { subject to } \\
& (A+\Delta A)+\mu_{i}(E+\Delta E) \quad \text { is singular for } i=1, \ldots, d .
\end{aligned}
$$

To formulate an optimization problem equivalent to (2.2), we introduce the functional

$$
G_{\varepsilon}(\Delta, \Theta)=\sum_{i=1}^{d}\left|\lambda_{i}(\Delta, \Theta)\right|^{2}
$$

where $\lambda_{i}(\Delta, \Theta)$ is the smallest (in absolute value) eigenvalue of the matrix $(A+\varepsilon \Delta)+$ $\mu_{i}(E+\varepsilon \Theta)$, for $\varepsilon>0$.

In order to minimize $G_{\varepsilon}(\Delta, \Theta)$, we construct two families of matrices $A+\varepsilon \Delta(t)$ and $E+\varepsilon \Theta(t)$, where $\Delta(t), \Theta(t) \in \mathbb{C}^{n, n}$ satisfies $\|[\Delta(t), \Theta(t)]\|_{F}=1$ and $G_{\varepsilon}(\Delta(t), \Theta(t))$ is 
a monotonically decreasing function of $t$ and $\lim _{t \rightarrow \infty} \Delta(t)=\Delta_{\infty}, \lim _{t \rightarrow \infty} \Theta(t)=\Theta_{\infty}$. These families are constructed such that $G_{\varepsilon}(\Delta, \Theta)$ is locally minimized over all such pairs $(\Delta, \Theta)$ of unit Frobenius norm.

REMARK 2.2. Alternatively, we can replace in (2.3) the smallest eigenvalue by the smallest singular value. The methods that we present below will work analogously for this modified cost functional. We compared both cost functionals in a few numerical experiments, where they worked similarly well.

REMARK 2.3. The choice of $\mu_{1}, \ldots, \mu_{d}$ may have an influence on the numerical results, but this did not appear to be a critical issue in our numerical experiments. We had good experience with the choice $\mu_{k}=r e^{2 \pi k \mathbf{i} / d}$ with a radius $r$ that is of the magnitude of $\|E\| /\|A\|$.

2.1. The inner iteration, steepest descent. To minimize the cost functional (2.3) with respect to the Frobenius norm, we use as inner iteration a steepest descent method and extend the ideas developed in $[8,9,10]$.

If $G_{\varepsilon}(\Delta, \Theta)>0$, then the derivatives $\dot{\Delta}, \dot{\Theta}$ are chosen in the direction that gives the maximum possible decrease of $G_{\varepsilon}(\Delta, \Theta)$. Here, to satisfy the norm constraint $\|[\Delta(t), \Theta(t)]\|_{F} \equiv 1$, we have to impose

$$
\operatorname{Re}\langle[\Delta, \Theta],[\dot{\Delta}, \dot{\Theta}]\rangle=0 .
$$

By Lemma 2.1, for a simple eigenvalue $\lambda(t)=r(t) \mathrm{e}^{\mathrm{i} \theta(t)}$ of the matrix-valued function $A+\varepsilon \Delta(t)+\mu(E+\varepsilon \Theta(t))$, with associated left and right eigenvectors $y(t)$ and $x(t)$, respectively, we have (omitting the dependence on $t$ )

$$
\begin{aligned}
\frac{1}{2} \frac{d}{d t}|\lambda|^{2} & =\operatorname{Re}(\bar{\lambda} \dot{\lambda})=\operatorname{Re}\left(\bar{\lambda} \varepsilon \frac{y^{H}(\dot{\Delta}+\mu \dot{\Theta}) x}{y^{H} x}\right) \\
& =\varepsilon \operatorname{Re}\left(\frac{(\lambda y)^{H}(\dot{\Delta}+\mu \dot{\Theta}) x}{y^{H} x}\right) \\
& =\varepsilon r \operatorname{Re}\left(\frac{y^{H}(\dot{\Delta}+\mu \dot{\Theta}) x}{\mathrm{e}^{\mathbf{i} \theta} y^{H} x}\right) .
\end{aligned}
$$

In the following we shall always impose a scaling of the eigenvectors $y$ and $x$ by

$$
\|y\|_{2}=\|x\|_{2}=1, \quad y^{H} x=\left|y^{H} x\right| \mathrm{e}^{-\mathbf{i} \theta}
$$

(using $\theta=0$ if $\lambda=0$ ) which makes the denominator of (2.4) real and positive (note that $\left|y^{H} x\right| \neq 0$ since $\lambda$ is assumed to be simple). Thus, with this normalization we have

$$
\frac{d}{d t}|\lambda|^{2}=2 \varepsilon \alpha \operatorname{Re}\left\langle y x^{H}, \dot{\Delta}+\mu \dot{\Theta}\right\rangle, \quad \text { with } \alpha=\frac{r}{\left|y^{H} x\right|},
$$

so that $\alpha$ is well defined by the simplicity assumption and $\alpha \neq 0$ if $\lambda \neq 0$.

Assuming (which is a generic property) that the smallest eigenvalue $\lambda_{i}$ of $A+$ $\varepsilon \Delta(t)+\mu_{i}(E+\varepsilon \Theta(t))$ is simple for all $i$, we obtain

$$
\frac{d}{d t} G_{\varepsilon}(\Delta, \Theta)=2 \varepsilon\left(\operatorname{Re}\left\langle\sum_{i=1}^{d} \alpha_{i} y_{i} x_{i}^{H}, \dot{\Delta}\right\rangle+\operatorname{Re}\left\langle\sum_{i=1}^{d} \alpha_{i} \bar{\mu}_{i} y_{i} x_{i}^{H}, \dot{\Theta}\right\rangle\right),
$$


with $\alpha_{i}=\frac{\left|\lambda_{i}\right|}{\left|y_{i}^{H} x_{i}\right|}$. Let

$$
K=\sum_{i=1}^{d} \alpha_{i} y_{i} x_{i}^{H}, \quad L=\sum_{i=1}^{d}\left(\alpha_{i} \bar{\mu}_{i}\right) y_{i} x_{i}^{H}
$$

Then the optimal steepest descent direction $[Z, W]=[\dot{\Delta}, \dot{\Theta}]$ for $G_{\varepsilon}(\Delta, \Theta)$ is determined by the following optimization problem:

$$
\begin{aligned}
{\left[Z_{*}, W_{*}\right]=} & \arg \min _{Z, W \in \mathbb{C}^{n, n}} \operatorname{Re}(\langle K, Z\rangle+\langle L, W\rangle) \\
& \text { subject to } \operatorname{Re}\langle[\Delta, \Theta],[Z, W]\rangle=0 \text { and }\|[Z, W]\|_{F}=1,
\end{aligned}
$$

where the normalization $\|[Z, W]\|_{F}=1$ guarantees uniqueness under the assumption that $\lambda_{i}$ is simple.

The optimum in (2.9) is characterized by the following lemma.

Lemma 2.4. Let $\Delta, \Theta \in \mathbb{C}^{n, n}$ with $\|[\Delta, \Theta]\|_{F}=1$, and let $K, L \in \mathbb{C}^{n, n}$ be such that $[K, L]$ is not proportional to $[\Delta, \Theta]$. Then the solution of the optimization problem (2.9) is given by

$$
\kappa[Z, W]=-[K, L]+\eta[\Delta, \Theta],
$$

where $\eta=\operatorname{Re}\langle[\Delta, \Theta],[K, L]\rangle$ and $\kappa$ is the Frobenius norm of the right hand side.

Proof. The result follows by noting that the expression in (2.10) is the orthogonal projection of $[K, L]$ onto the vector space $\{[Z, W]: \operatorname{Re}\langle[\Delta, \Theta],[Z, W]\rangle=0\}$.

Using Lemma 2.4, we obtain a gradient system for $G_{\varepsilon}(\Delta, \Theta)$ with initial data $\left[\Delta_{0}, \Theta_{0}\right]$ of unit Frobenius norm, which is given by

$$
\begin{aligned}
& \dot{\Delta}=-K+\eta \Delta, \\
& \dot{\Theta}=-L+\eta \Theta,
\end{aligned}
$$

where $K$ and $L$ are defined by $(2.8)$ and $\eta=\operatorname{Re}\langle\Delta, K\rangle+\operatorname{Re}\langle\Theta, L\rangle$.

The following result is an immediate consequence of (2.11).

THEOREM 2.5. Let $\Delta(t), \Theta(t)$ with $\|[\Delta(t), \Theta(t)]\|_{F}=1$ satisfy the system of differential equations (2.11). If $\lambda_{i}(t)$ is a simple eigenvalue of $A+\varepsilon \Delta(t)+\mu_{i}(E+$ $\varepsilon \Theta(t))$, then

$$
\frac{d}{d t} G_{\varepsilon}(\Delta(t), \Theta(t)) \leq 0
$$

Proof. The result follows directly from Lemmas 2.1 and 2.4. $\quad$

REMARK 2.6. At a stationary point of $G_{\varepsilon}$, if $G_{\varepsilon}>0$, we have that $[\Delta, \Theta]$ is a real multiple of $[K, L]$, unless $[K, L]=0$. However, generically we have $[K, L] \neq 0$; in fact it is immediate that

$$
\operatorname{trace}(K)=\sum_{i=1}^{d} \lambda_{i} \quad \text { and } \quad \operatorname{trace}(L)=\sum_{i=1}^{d} \bar{\mu}_{i} \lambda_{i},
$$

which are both generically different from zero (note that the set of real vectors $\left(\operatorname{Re}\left(z_{1}\right), \operatorname{Im}\left(z_{1}\right), \ldots, \operatorname{Re}\left(z_{d}\right), \operatorname{Im}\left(z_{d}\right)\right)$ such that $\sum_{1 \leq i \leq d} \operatorname{Re}\left(z_{i}\right)=\sum_{1 \leq i \leq d} \operatorname{Im}\left(z_{i}\right)=0$ 
is a subspace of $\mathbb{R}^{2 d}$ of dimension $2 d-2$ ). Consequently $K$ and $L$ generically do not vanish.

REMARK 2.7. Integrating the system (2.11) will usually lead us (by monotonicity) to a local minimum which is not necessarily global. In order to increase the robustness of the approach it is useful to compute several trajectories starting from different initial data.

2.2. The outer iteration, updating $\varepsilon$. For every $\varepsilon>0$, the gradient system (2.11) yields a stationary point $[\Delta(\varepsilon), \Theta(\varepsilon)]$ of unit Frobenius norm that is a (local) minimum of $G_{\varepsilon}$. To update the parameter $\varepsilon$ aiming at $G_{\varepsilon}(\Delta(\varepsilon), \Theta(\varepsilon))=0$, we propose an outer iteration in form of a Newton-like method. The method is constructed to approach, from the left-hand side, the value $\varepsilon^{\star}>0$ such that $G_{\varepsilon^{\star}}\left(\Delta\left(\varepsilon^{\star}\right), \Theta\left(\varepsilon^{\star}\right)\right)=0$ and $G_{\varepsilon}(\Delta(\varepsilon), \Theta(\varepsilon))>0$ for $\varepsilon<\varepsilon^{\star}$.

In general we expect that for a given $\varepsilon<\varepsilon^{\star}$ all eigenvalues $\lambda_{i}$ are different from zero and simple. If so $G_{\varepsilon}(\Delta(\varepsilon), \Theta(\varepsilon))$ would be a smooth function of $\varepsilon$ and we may exploit its regularity by determining an exact expression for its derivative, to be used in the fast outer iteration we are going to present. Otherwise we should make use of a bisection technique in order to approach $\varepsilon^{\star}$.

Assumption 2.8. For $\varepsilon<\varepsilon^{\star}$, the smallest eigenvalue $\lambda_{i}(\varepsilon)$ of the matrix $A+$ $\varepsilon \Delta(\varepsilon)+\mu_{i}(E+\varepsilon \Theta(\varepsilon))$ is simple and different from zero, and the functions $\Delta(\varepsilon), \Theta(\varepsilon)$, and $\lambda_{i}(\varepsilon), i=1, \ldots, d$ are sufficiently smooth with respect to $\varepsilon$ (at least near $\varepsilon^{\star}$ ).

Assumption 2.8 guarantees that the eigenvalue $\lambda_{i}(\varepsilon)$ of $A+\varepsilon \Delta(\varepsilon)+\mu_{i}(E+\varepsilon \Theta(\varepsilon))$ is a smooth function of $\varepsilon$ in a left neighborhood of $\varepsilon^{\star}$. This property is exploited in the following result which provides an explicit and easily computable expression for the derivative of $G_{\varepsilon}(\Delta(\varepsilon), \Theta(\varepsilon))$ with respect to $\varepsilon$. Here, $K(\varepsilon)$ and $L(\varepsilon)$ are the matrices (2.8) that correspond to the eigenvalues $\lambda_{i}(\varepsilon)$. Both $K(\varepsilon)$ and $L(\varepsilon)$ are generically nonzero by Remark 2.6.

TheOREM 2.9. Consider a regular pencil $(A, E)$ with $A, E \in \mathbb{C}^{n, n}$ and a perturbed pencil $(A+\varepsilon \Delta(\varepsilon), E+\varepsilon \Theta(\varepsilon))$ that has the following properties:

i) $\varepsilon \in\left(0, \varepsilon^{\star}\right)$, so that $G_{\varepsilon}(\Delta(\varepsilon), \Theta(\varepsilon))>0$;

ii) Assumption 2.8 holds.

Then,

iii) $[K(\varepsilon), L(\varepsilon)] \neq 0$.

$$
\frac{d G_{\varepsilon}(\Delta(\varepsilon), \Theta(\varepsilon))}{d \varepsilon}=-\|[K(\varepsilon), L(\varepsilon)]\|_{F}<0 .
$$

Proof. Since by assumption $\lambda(\varepsilon)$ is simple, we can employ the derivative formula (Lemma 2.1) and, denoting the derivative $\frac{d}{d \varepsilon}$ by ', we obtain

$$
\frac{d}{d \varepsilon} \lambda(\varepsilon)=\frac{y(\varepsilon)^{H}\left(\Delta(\varepsilon)+\varepsilon \Delta^{\prime}(\varepsilon)+\mu \Theta(\varepsilon)+\mu \varepsilon \Theta^{\prime}(\varepsilon)\right) x(\varepsilon)}{y(\varepsilon)^{H} x(\varepsilon)} .
$$

Applying this similarly in (2.6) we obtain

$$
\begin{aligned}
\frac{1}{2} \frac{d}{d \varepsilon}|\lambda(\varepsilon)|^{2} & =\operatorname{Re}\left(\bar{\lambda}(\varepsilon) \frac{d}{d \varepsilon} \lambda(\varepsilon)\right) \alpha(\varepsilon) \\
& =\alpha(\varepsilon) \operatorname{Re}\left(\left\langle y(\varepsilon) x(\varepsilon)^{H}, \Delta(\varepsilon)+\varepsilon \Delta^{\prime}(\varepsilon)+\mu \Theta(\varepsilon)+\mu \varepsilon \Theta^{\prime}(\varepsilon)\right\rangle\right) .
\end{aligned}
$$

Therefore,

$$
\frac{d}{d \varepsilon} G_{\varepsilon}(\Delta(\varepsilon), \Theta(\varepsilon))=\operatorname{Re}\left\langle K(\varepsilon), \Delta(\varepsilon)+\varepsilon \Delta^{\prime}(\varepsilon)\right\rangle+\operatorname{Re}\left\langle L(\varepsilon), \Theta(\varepsilon)+\varepsilon \Theta^{\prime}(\varepsilon)\right\rangle .
$$


To prove the assertion, we show that

$$
\operatorname{Re}\left\langle K(\varepsilon), \Delta^{\prime}(\varepsilon)\right\rangle+\operatorname{Re}\left\langle L(\varepsilon), \Theta^{\prime}(\varepsilon)\right\rangle=0 .
$$

The minimality property of $G_{\varepsilon}(\Delta(\varepsilon), \Theta(\varepsilon))$ implies that

$$
\operatorname{Re}\left\langle K(\varepsilon), \Delta^{\prime}(\varepsilon)\right\rangle+\operatorname{Re}\left\langle L(\varepsilon), \Theta^{\prime}(\varepsilon)\right\rangle \geq 0 .
$$

Now suppose that for some $\varepsilon_{0}$, this inequality would actually be strict. Consider $[\widetilde{\Delta}(\varepsilon), \widetilde{\Theta}(\varepsilon)]$ of unit norm such that both $\left(\widetilde{\Delta}\left(\varepsilon_{0}\right), \widetilde{\Theta}\left(\varepsilon_{0}\right)\right)=\left(\Delta\left(\varepsilon_{0}\right), \Theta\left(\varepsilon_{0}\right)\right)$ as well as $\left(\widetilde{\Delta}^{\prime}\left(\varepsilon_{0}\right), \widetilde{\Theta}^{\prime}\left(\varepsilon_{0}\right)\right)=\left(-\Delta^{\prime}\left(\varepsilon_{0}\right),-\Theta^{\prime}\left(\varepsilon_{0}\right)\right)$ holds. Then, for all $\varepsilon$ sufficiently close to $\varepsilon_{0}$, we would have the corresponding inequality $G_{\varepsilon}(\widetilde{\Delta}(\varepsilon), \widetilde{\Theta}(\varepsilon))<G_{\varepsilon}(\Delta(\varepsilon), \Theta(\varepsilon))$. This, however, contradicts the minimality of $(\Delta(\varepsilon), \Theta(\varepsilon))$ and hence $(2.14)$ holds.

By assumption iii), extremizers of $G_{\varepsilon}(\Delta, \Theta)$, which are stationary points of (2.11), have the property that $\Delta(\varepsilon)$ is proportional to $K(\varepsilon)$ and $\Theta(\varepsilon)$ is proportional to $L(\varepsilon)$ with the factor $\eta=\operatorname{Re}\langle\Delta(\varepsilon), K(\varepsilon)\rangle+\operatorname{Re}\langle\Theta(\varepsilon), L(\varepsilon)\rangle$. Together with (2.13) and (2.14), this concludes the proof.

The derivative formula in Theorem 2.9 allows us to construct a quadratically convergent Newton-like method to compute an approximation to the root $\varepsilon^{\star}$ of $g(\varepsilon)=$ $G_{\varepsilon}(\Delta(\varepsilon), \Theta(\varepsilon))$, which can be expanded as

$$
g(\varepsilon) \approx \chi\left(\varepsilon^{\star}-\varepsilon\right)^{2}
$$

and can be estimated by the knowledge of $g(\varepsilon)$ and $g^{\prime}(\varepsilon)$ (via Theorem 2.9) for $\varepsilon<\varepsilon^{\star}$.

Solving with respect to $\varepsilon$ gives

$$
\varepsilon^{\star} \approx \varepsilon-2 \frac{g(\varepsilon)}{g^{\prime}(\varepsilon)}, \quad \chi \approx \frac{g^{\prime}(\varepsilon)^{2}}{4 g(\varepsilon)} .
$$

For $\varepsilon=\varepsilon_{k}<\varepsilon^{\star}$, we obtain the Newton iteration

$$
\varepsilon_{k+1}=\varepsilon_{k}-2 \frac{g\left(\varepsilon_{k}\right)}{g^{\prime}\left(\varepsilon_{k}\right)},
$$

and get a locally quadratically convergent iteration from the left (if instead $\varepsilon_{k}>\varepsilon^{\star}$ we should use bisection, which would give a linear reduction of the error from the right).

2.3. Numerical examples. To illustrate the inner-outer iteration described in the last subsections we present some numerical examples. We first consider a problem with fixed $E$ so that the gradient system is simplified to

$$
\dot{\Delta}=-K+\eta \Delta,
$$

where $K$ is defined in (2.8) and $\eta=\operatorname{Re}\langle\Delta, K\rangle$.

All examples have dimension 3 and we use $\mu_{\ell}=\mathrm{e}^{\mathrm{i} \ell \pi / 2}, \ell=1, \ldots, 4$ as evaluation points to check the singularity.

Example 2.10. Consider the pencil $(A, E)$ with

$$
A=\left[\begin{array}{rrr}
0 & 0.0400 & 0.8900 \\
0.1500 & -0.0200 & 0 \\
0.9200 & 0.1100 & 0.0600
\end{array}\right], \quad E=\left[\begin{array}{lll}
0 & 0 & 0 \\
0 & 0 & 1 \\
0 & 1 & 0
\end{array}\right]
$$


By repeated numerical integration of (2.15), perturbing only $A$, we compute an upper bound $d_{A}(A, E) \approx \varepsilon^{\star}=0.1357$ and

$$
\begin{aligned}
& A+\varepsilon^{\star} \Delta=\left[\begin{array}{rr}
-0.0000000001 & -0.0542959187+0.0000017493 \mathbf{i} \\
0.0568651065+0.0000071780 \mathbf{i} & 0.0068225209+0.0000025925 \mathbf{i} \\
0.9231408234-0.0000304036 \mathbf{i} & 0.1128955997+0.0002292806 \mathbf{i}
\end{array}\right. \\
& \left.\begin{array}{l}
0.8814329362-0.0001686901 \mathbf{i} \\
0.0059287782+0.0002106238 \mathbf{i} \\
0.0615119378+0.0000843775 \mathbf{i}
\end{array}\right] \text {. }
\end{aligned}
$$

We remark that the singular pencil $\left(A+\varepsilon^{\star} \Delta, E\right)$ has no common null vectors and the extremal perturbation $\Delta$ has full rank.

EXAMPLE 2.11. For

$$
A=\left[\begin{array}{rrr}
-1.79 & 0.10 & -0.60 \\
0.84 & -0.54 & 0.49 \\
-0.89 & 0.30 & 0.74
\end{array}\right], \quad E=\left[\begin{array}{lll}
0 & 0 & 0 \\
0 & 0 & 1 \\
0 & 1 & 0
\end{array}\right],
$$

perturbing only $A$, by repeated numerical integration of (2.15), we compute an upper bound $d_{A}(A, E) \approx \varepsilon^{\star}=1.8907$ and a real perturbed matrix

$$
A+\varepsilon^{\star} \Delta=\left[\begin{array}{rrr}
0.0000000005 & 0.0000000006 & 0.0000000011 \\
0.8213322643 & -0.5362641604 & 0.4731712699 \\
-0.8893596899 & 0.3006536120 & 0.7400519795
\end{array}\right] .
$$

In this case the singular pencil $\left(A+\varepsilon^{\star} \Delta, E\right)$ has a common left null vector $\left[\begin{array}{ccc}1 & 0 & 0\end{array}\right]^{T}$ and the extremal perturbation $\Delta$ (of unit Frobenius norm) has a dominant singular value $\sigma_{1} \approx 0.999998$ which indicates that the exact extremal perturbation may have rank one (see Section 3 ).

In the first two examples we have only perturbed the matrix $A$, in the next two examples we consider the pencils from the first two examples but allow perturbations in both $E$ and $A$.

EXAMPLE 2.12. Consider again the pencil of Example 2.10. Allowing perturbations in both matrices and using repeated numerical integration of (2.11), we compute an upper bound $d(A, E) \approx \varepsilon^{\star}=0.1193$ and perturbed matrices (displayed to 10 digits)

$$
A+\varepsilon^{\star} \Delta=\left[\begin{array}{rrr}
0.0001475316 & -0.0236092412 & 0.8794938198 \\
0.0820097750 & 0.0105557540 & 0.0146194487 \\
0.9192560915 & 0.1211438423 & 0.0587793566
\end{array}\right]
$$

and

$$
E+\varepsilon^{\star} \Theta=\left[\begin{array}{rrr}
0.0000022028 & 0.0055760367 & 0.0115091616 \\
-0.0049781822 & 0.0612871149 & 1.0094299281 \\
0.0120766466 & 0.9993449498 & -0.0001655503
\end{array}\right] .
$$

Note that in this case (up to four digits) $\|\Delta\|_{F}=0.84043$ and $\|\Theta\|_{F}=0.54191$ so that the matrix $A$ has been perturbed more significantly than $E$. The singular pencil $\left(A+\varepsilon^{\star} \Delta, E+\varepsilon^{\star} \Theta\right)$ is closer to $(A, E)$ than the one computed in Example 2.10 and has still no common null vector. Again the extremal perturbations $\Delta, \Theta$ have full rank and in this case they are real. 
EXAMPLE 2.13. Consider again the pencil of Example 2.11. Allowing perturbations in both matrices and using repeated numerical integration of (2.11), we compute an upper bound $d(A, E) \approx \varepsilon^{\star}=1.065$ and perturbed matrices (to 10 digits)

$$
\begin{aligned}
& A+\varepsilon^{\star} \Delta=\left[\begin{array}{c}
-1.5597093229+0.0021610076 \mathbf{i} \\
1.0048046874-0.0445127183 \mathbf{i} \\
-0.8924856932-0.0641270083 \mathbf{i}
\end{array}\right. \\
& \left.\begin{array}{cc}
0.3260473995+0.0966265200 \mathbf{i} & -0.7591513321+0.0449684360 \mathbf{i} \\
-0.2219278843-0.0447293570 \mathbf{i} & 0.4034298635-0.0585117306 \mathbf{i} \\
0.3083824662-0.0641142654 \mathbf{i} & 0.8035571677+0.0366925210 \mathbf{i}
\end{array}\right] \text {, } \\
& E+\varepsilon^{\star} \Theta=\left[\begin{array}{l}
0.1399454874-0.0182763461 \mathbf{i} \\
0.2452949522+0.1507937938 \mathbf{i} \\
0.0876482905+0.2170776953 \mathbf{i}
\end{array}\right. \\
& 0.2658606960-0.1821019435 \mathbf{i} \quad-0.2014892070-0.0418178132 \mathbf{i}] \\
& -0.2351476376+0.0310916192 \mathbf{i} \quad 0.5770786070+0.0072381773 \mathbf{i} \\
& 0.5426711711-0.0549700013 \mathbf{i} \quad-0.2920820819-0.0020714181 \mathbf{i}]
\end{aligned}
$$

In this case (rounded to four digits) $\|\Delta\|_{F}=0.5124$ and $\|\Theta\|_{F}=0.8588$ so that the matrix $E$ has been perturbed more significantly than $A$. Again the singular pencil $\left(A+\varepsilon^{\star} \Delta, E+\varepsilon^{\star} \Theta\right)$ is closer to $(A, E)$ than that of Example 2.11, but in this case there is no common null vector. The extremal perturbations $\Delta, \Theta$ have full rank and in this case the extremal perturbations $\Delta, \Theta$ are non-real.

An extensive numerical investigation suggests that in the case of singular pencils with a common null vector the extremal perturbations $\Delta, \Theta$ have rank one. This will be proved rigorously below.

2.4. Real perturbations. In this subsection we extend the approach to the case where the matrices $A, E$ are real and the perturbations $\Delta A, \Delta E$ are required to be real as well. In this case we compute

$$
d^{\mathbb{R}}(A, E)=\min \{\|[\Delta A, \Delta E]\|,:(A+\Delta A, E+\Delta E) \text { is real and singular }\} .
$$

We can simply extend Lemma 2.4 and replace the matrices $K, L$ by $\operatorname{Re}(K)$ and $\operatorname{Re}(L)$. This yields the system of ODEs

$$
\left\{\begin{array}{l}
\dot{\Delta}=-\operatorname{Re}(K)+\eta \Delta \\
\dot{\Theta}=-\operatorname{Re}(L)+\eta \Theta
\end{array}\right.
$$

where $K$ and $L$ are defined by (2.8) and $\eta=\langle\Delta, \operatorname{Re}(K)\rangle+\operatorname{Re}\langle\Theta, \operatorname{Re}(L)\rangle$.

The whole theory remains unaltered. Let us consider an example.

Example 2.14. Consider again the pencil from Example 2.10 perturbing only $A$. By restricting to real perturbations, we obtain an upper bound $d_{A}^{\mathbb{R}}(A, E) \approx \varepsilon^{\star}=$ 0.1366 and the perturbed matrix is

$$
A+\varepsilon^{\star} \Delta=\left[\begin{array}{rrr}
0.0000000549 & -0.0540181921 & 0.8756835927 \\
0.0566705179 & 0.0068457984 & 0.0108125955 \\
0.9186800977 & 0.1180892075 & 0.0599641664
\end{array}\right] .
$$

Again the perturbed matrices $A+\varepsilon^{\star} \Delta, E$ have no common null vector. 
2.5. Comparison with purely algebraic approaches. The new approach is based on optimization methods for differential equations. The question arises whether the approximations obtained via this approach are superior to those obtained in a purely linear algebra fashion. In general these different approaches are hard to compare, since they proceed via completely different optimization methods. Except for special cases, see $[2,21]$, no proof is available that the true distance has been obtained. However, extensive numerical tests indicate that the differential equation approach achieves smaller bounds for the distance to singularity in all cases.

The easiest of the linear algebra approaches (which is not using an optimization) is to compute the generalized Schur form $\left(U^{H} E V, U^{H} A V\right)=(S, T)$ of the pair $(A, E)$ with upper triangular matrices $S, T$ and then to create a pair of zero diagonal elements in the upper triangular matrices $S$ and $T$.

Example 2.15. Consider the pair $(A, E)$ from Example 2.10. Applying the QZ algorithm, see e.g. [6], to this pair gives the generalized Schur form with

$$
\begin{aligned}
S & =\left[\begin{array}{cccc}
0 & -0.978747365727621 & 0.205069729802429 \\
0 & 0.205069729802429 & 0.978747365727621 \\
0 & 0 & -0.000000000000001
\end{array}\right], \\
T & =\left[\begin{array}{ccc}
0.932148056909416 & -0.102583031602284 & 0.063888312298283 \\
0 & 0.036969147238194 & -0.011326399756439 \\
0 & 0 & 0.890898422941695
\end{array}\right]
\end{aligned}
$$

Considering the third diagonal element of $S$ to be zero, we see that an upper bound for the smallest (real and even rank-one) perturbation in $T$ that makes the pencil singular is of size $\approx 0.8909$, while the smallest perturbation in both matrices is obtained by perturbing the second diagonal element in both $S$ and $T$ to zero, which is of order 0.2084 in Frobenius norm. Both perturbations are larger than the minimal perturbations obtained by the ODE approach.

ExAmple 2.16. Consider the following example from [2] with matrices

$$
A=U^{T}\left[\begin{array}{ccc}
1 & 0 & 0 \\
0 & .0001 & 0 \\
0 & 0 & 1
\end{array}\right] V, \quad E=U^{T}\left[\begin{array}{lll}
0 & 1 & 0 \\
0 & 0 & 1 \\
0 & 0 & 0
\end{array}\right] V
$$

with real orthogonal matrices $U, V$. The smallest perturbation that makes the pencil singular, perturbing both $E, A$ or only $A$ is real, of rank one and of size .0001 .

The ODE approach correctly yields a closest singular pair at a distance approximately .0001 for different choices of $U$ and $V$.

Example 2.17. Consider next the following example of an $8 \times 8$ matrix pencil arising from the model of a two-dimensional, three-link mobile manipulator from $[2$, Example 14].

$$
A=\left[\begin{array}{ccc}
\mathrm{O} & \mathrm{I}_{3} & \mathrm{O} \\
-K_{0} & -D_{0} & F_{0}^{\mathrm{T}} \\
F_{0} & \mathrm{O} & \mathrm{O}
\end{array}\right], E=\left[\begin{array}{ccc}
\mathrm{I}_{3} & \mathrm{O} & \mathrm{O} \\
\mathrm{O} & M_{0} & \mathrm{O} \\
\mathrm{O} & \mathrm{O} & \mathrm{O}
\end{array}\right]
$$


with

$$
\begin{aligned}
& M_{0}=\left[\begin{array}{rrr}
18.7532 & -7.94493 & 7.94494 \\
-7.94493 & 31.8182 & -26.8182 \\
7.94494 & -26.8182 & 26.8182
\end{array}\right], D_{0}=\left[\begin{array}{rrr}
-1.52143 & -1.55168 & 1.55168 \\
3.22064 & 3.28467 & -3.28467 \\
-3.22064 & -3.28467 & 3.28467
\end{array}\right] \text {, } \\
& K_{0}=\left[\begin{array}{rrr}
67.4894 & 69.2393 & -69.2393 \\
69.8124 & 1.68624 & -1.68617 \\
-69.8123 & -1.68617 & -68.2707
\end{array}\right], F_{0}=\left[\begin{array}{lll}
1 & 0 & 0 \\
0 & 0 & 1
\end{array}\right] \text {. }
\end{aligned}
$$

An application of the method presented in this paper gives as result the bound $d(A, E) \approx 0.011$, which is consistent with the best result given in [2]. Here the closest singular pair does not have any common left or right null vector. The modulus of $G_{\varepsilon}(\Delta(\varepsilon), \Theta(\varepsilon)$ ) is plotted (in logarithmic scale) in Figure 2.1.

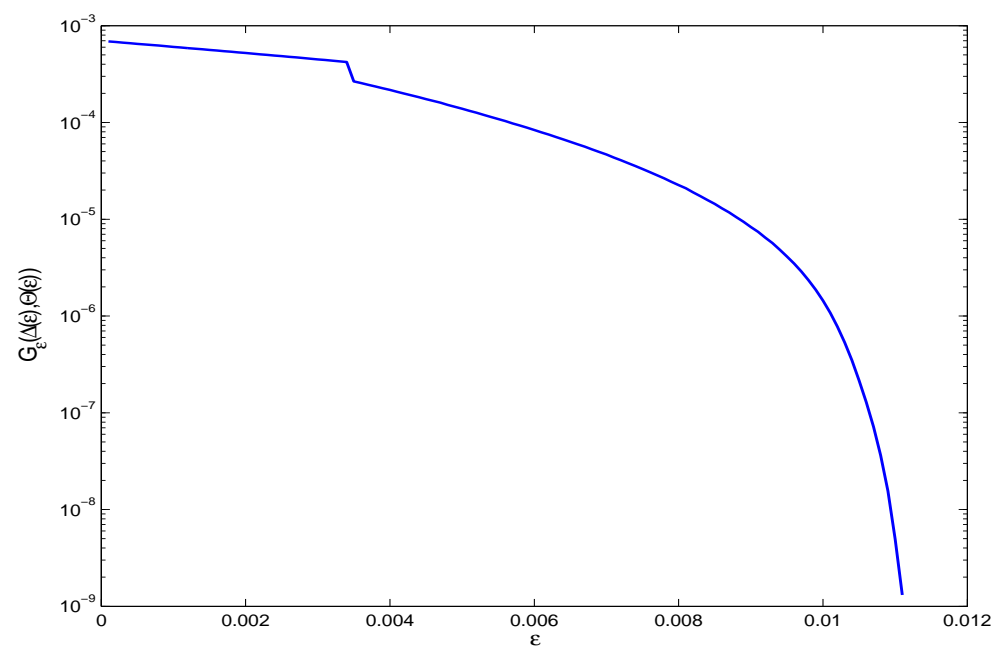

FIG. 2.1. The function $\varepsilon \rightarrow G_{\varepsilon}(\Delta(\varepsilon), \Theta(\varepsilon))$ for the illustrative example 2.17

We have seen in this section that with the ODE approach we are able to compute approximations from above for the distance to singularity, perturbing both matrices $A, E$ or only $A$ and we can even deal with the real case analogously.

It cannot be guaranteed that the optimization approach reaches a global minimum. However, starting from different initial values, the method has the option to find several local minima. As the examples demonstrate, the minimum may not be achieved by a perturbation where the perturbed matrices have a common null vector. However, if we make it a requirement that we are only looking for a smallest perturbation with this property, then the situation changes drastically. This will be discussed in the next section.

3. Distance to singular pencils with common null vectors: the case of fixed $E$. In this section we consider the ODE-based approach for the minimization problem of finding a nearby pencil with a common right null vector. A common left null vector is simply obtained by applying the same procedure to the pair $\left(A^{H}, E^{H}\right)$. We first discuss extensively the case that only the matrix $A$ is perturbed and then extend the approach to the perturbation of both matrices in the next section. In both cases we prove that the smallest perturbations which make the pencil singular, have rank one. 
3.1. Simple upper and lower bounds for $d_{A}^{0}(A, E)$. Let us first discuss purely algebraic techniques to construct some upper and lower bounds.

The simplest lower bound is given by the distance to singularity of $A$, which in the unstructured case is given by the smallest singular value $\sigma_{n}(A)$. This provides the lower bound

$$
\sigma_{n}(A) \leq d_{A}^{0}(A, E)
$$

To get an upper bound for the distance to singularity we can employ the generalized singular value decomposition of the pair $(A, E)$, see [6]. We have the following Lemma.

Lemma 3.1. Let $A, E \in \mathbb{C}^{n, n}$ with $E$ singular and $(A, E)$ regular. Let $X A V=C$, $X E U=D$ be the generalized singular value decomposition with $U, V$ unitary, $X$ invertible, and $C=\operatorname{diag}\left(C_{1}, C_{2}\right), D=\operatorname{diag}\left(D_{1}, 0\right)$, diagonal, $D_{1}$ invertible, and the diagonal elements of $C_{2}$ are ordered in decreasing absolute value. Then the matrices $A-\left(e_{n}^{T} C_{2} e_{n}\right) X^{-1} e_{n} e_{n}^{T} U^{H}, E$ have a common right null vector $U e_{n}$.

Proof. The proof is straightforward, since by construction

$$
X\left(A-\left(e_{n}^{T} C_{2} e_{n}\right) X^{-1} e_{n} e_{n}^{T} U^{H}\right) U e_{n}=0, \quad X E U \mathrm{e}_{n}=0,
$$

which proves the result.

Lemma 3.1 gives a numerically computable rank-one perturbation to $A$ that creates a common null vector, which even is real in the real case. This clearly gives a simple upper bound for the distance to singularity. This perturbation would actually be minimal in Frobenius norm if the matrix $X$ were unitary, which however is usually not the case. A sufficient condition for $X$ to be unitary is that $E^{H} A=A^{H} E$ and $E A^{H}=A E^{H}$, which is however, a very strong requirement.

Another upper bound can be obtained from the Jordan canonical form

$$
A=T J T^{-1} \text {. }
$$

Consider any Jordan block with eigenvalue $\lambda_{m}$ and replace the first diagonal element in this block by zero (calling the resulting matrix $\hat{J}_{m}$ ) and replace the associated eigenvector $x_{m}$ in the associated column of $T$ by its orthogonal projection onto the kernel of $E$ leading to a matrix $\hat{T}_{m}$. This projection is obtained by setting $\hat{x}_{m}=$ $x_{m}-V_{2} V_{2}^{H} x_{m}$, where $E=U \Sigma V^{H}$ is the singular value decomposition of $E$ and the columns of $V_{2}$ span the kernel of $E$. If $\hat{x}_{m}$ is linearly independent to the remaining columns of $T$ then the new pair $(E, \hat{A})=\left(E, \hat{T}_{m} \hat{J}_{m} \hat{T}_{m}^{-1}\right)$ is singular. Then, minimizing $\left\|A-\hat{A}_{m}\right\|$ by going through all eigenvalues of $A$, we obtain the upper bound

$$
d_{A}^{0}(A, E) \leq\left\|A-\hat{A}_{m^{*}}\right\|,
$$

where $m^{*}$ is the index of the eigenvalue where the bound is minimized. This upper bound is of limited practical value, since its computation requires the Jordan canonical form which is usually not numerically computable. Using instead the ordered Schur form, see $[6]$,

$$
A=Q S Q^{H}
$$

with $Q$ unitary and $S$ upper triangular, we have that the first column of $Q$ forms an eigenvector and we can apply the same approach of setting the eigenvalue to 0 and replace the eigenvector by its orthogonal projection onto the kernel of $E$, to obtain 
singular pencil. Using eigenvalue reordering, see [6], we can reorder the eigenvalues one by one to the top and apply the projection in the same way and then minimize over all possible eigenvalues. This gives a numerically computable upper bound realized by a rank-one perturbation. Usually this approach cannot be applied to obtain a real perturbation if the eigenvalues are non-real. Further bounds obtained from a purely algebraic approach are derived in [2].

A third upper bound is obtained from the singular value decomposition

$$
U^{H}\left[\begin{array}{l}
E \\
A
\end{array}\right] V=\left[\begin{array}{l}
\Sigma \\
0
\end{array}\right],
$$

with $\Sigma=\operatorname{diag}\left(\sigma_{1}, \ldots, \sigma_{n}\right)$ and

$$
U=\left[\begin{array}{ll}
U_{11} & U_{12} \\
U_{21} & U_{22}
\end{array}\right]
$$

with $U_{i, j} \in \mathbb{C}^{n, n}$. Then the pencil

$$
\left(A-\sigma_{n} U_{21} e_{n} e_{n}^{T} V^{H}, E-\sigma_{n} U_{11} e_{n} e_{n}^{T} V^{H}\right)
$$

is singular with common null vector $V e_{n}$, since

$$
U^{H}\left[\begin{array}{c}
E-\sigma_{n} U_{11} e_{n} e_{n}^{T} V^{H} \\
A-\sigma_{n} U_{21} e_{n} e_{n}^{T} V^{H}
\end{array}\right] V e_{n}=0 .
$$

This is, however, a perturbation in both matrices and it is a rank-one perturbation in each of the two.

Example 3.2. Consider the pencil from Example 2.11. In this case the perturbation obtained via the singular value decomposition in (3.1) is given by

$$
\Delta A=\left[\begin{array}{ccc}
-0.0851 & -0.2996 & 0.0397 \\
-0.0948 & -0.3335 & 0.0442 \\
-0.0132 & -0.0465 & 0.0062
\end{array}\right], \Delta E=\left[\begin{array}{ccc}
0.0000 & 0.0000 & -0.0000 \\
-0.0343 & -0.1208 & 0.0160 \\
0.2587 & 0.9105 & -0.1208
\end{array}\right],
$$

with $d(A, E)=1.0722$ which is larger than the bound 1.065 obtained by the ODE approach.

To obtain a rank-one perturbation in $A$ only, we consider the singular value decomposition of $E$,

$$
E=U\left[\begin{array}{cc}
\Sigma_{m} & 0 \\
0 & 0
\end{array}\right] V^{H}
$$

with $U \in \mathbb{C}^{n, n}$ and $V \in \mathbb{C}^{n, n}$ unitary and $\Sigma_{m} \in \mathbb{R}^{m, m}$ diagonal with positive diagonal entries. (Note that in the real case the factors are real.) Partition

$$
U^{H} A V=\left[\begin{array}{ll}
A_{11} & A_{12} \\
A_{21} & A_{22}
\end{array}\right]
$$

with $A_{11} \in \mathbb{C}^{m, m}$ accordingly, and let $A_{22}=U_{A} \Sigma_{A} V_{A}^{H}$ be another singular value decomposition, with $\Sigma_{A}=\operatorname{diag}\left(\xi_{1}, \ldots, \xi_{n-m}\right)$ diagonal entries. Let

$$
\tilde{U}:=U\left[\begin{array}{ll}
I_{m} & \\
& U_{A}
\end{array}\right], \tilde{V}:=V\left[\begin{array}{ll}
I_{m} & \\
& V_{A}
\end{array}\right]
$$


and set

$$
\Delta A=-\tilde{U}\left[\begin{array}{cc}
0 & A_{12} e_{n-m} e_{n-m}^{T} \\
0 & \xi_{n-m} e_{n-m} e_{n-m}^{T}
\end{array}\right] \tilde{V}^{H}
$$

Then by construction $x:=\tilde{V} e_{n}$ is a common null vector of $E$ and $A+\Delta A$, but we do not know whether this is of minimal Frobenius norm.

EXAMPLE 3.3. Consider again the pencil from Example 2.11. In this case the perturbation obtained via (3.2) is 2.1684 which is again larger than the bound 1.8907 obtained via the ODE approach.

In the following subsection we proceed again via the ODE approach.

3.2. An ODE-based approach for computing $d_{A}^{0}(A, E)$. In the following we assume that $E \in \mathbb{C}^{n, n}$ is singular, has rank $m<n$ and is not perturbed. As in the algebraic approach in the previous subsection, to reformulate the problem, we construct a perturbation so that $A+\Delta A$ has an eigenvector $x$ to the eigenvalue 0 in the kernel of $E$, so that

$$
(A+\Delta A+\mu E) x=0 \quad \text { for all } \mu \in \mathbb{C} .
$$

This leads to the following constrained minimization problem

$$
\begin{gathered}
\Delta A_{*}=\arg \min _{\Delta A \in \mathbb{C}^{n, n}}\|\Delta A\| \\
\text { subject to }(A+\Delta A) x=0 \quad \text { for some } x \in \mathbb{C}^{n}, x \neq 0 \\
\text { and } \quad E x=0,
\end{gathered}
$$

so that $d_{A}^{0}(A, E)=\left\|\Delta A_{*}\right\|$. This is a classical matrix nearness problem, see [14].

To employ an ODE based optimization approach, we make use of the restricted singular value decomposition $E=U \Sigma V^{H}$ with $U \in \mathbb{C}^{n, m}$ and $V \in \mathbb{C}^{n, m}$ unitary and $\Sigma_{m} \in \mathbb{R}^{m, m}$ diagonal with positive entries. Note that in the real case the factors are real.

In the inner iteration, for any fixed $\varepsilon$ we compute a (local) minimizer of the functional

$$
F_{\varepsilon}(\Delta)=\frac{1}{2}|\lambda(\Delta)|^{2}+\frac{1}{2}\left\|V^{H} x(\Delta)\right\|_{2}^{2},
$$

where $(x, \lambda)$ is an eigenvalue/eigenvector pair of $A+\varepsilon \Delta$ and $\|\Delta\|_{F}=1$.

The local minimizer is determined as the stationary point of the associated gradient system. In general this will not be a global minimizer, although this seems to be the case in all our experiments of small dimension (where we performed an investigation on a very large number of samples).

For the outer iteration, we consider a continuous branch of minimizers $\Delta(\varepsilon)$ and vary $\varepsilon$ iteratively by exploiting the knowledge of the exact derivative of $f(\varepsilon)=$ $F_{\varepsilon}(\Delta(\varepsilon))$ with respect to $\varepsilon$. We obtain superlinear convergence.

To derive the gradient system we make use of the following definition.

DeFinition 3.4. Let $M$ be a singular matrix with a simple zero eigenvalue. The group inverse (reduced resolvent) of $M$ is the unique matrix $G$ satisfying

$$
M G=G M, \quad G M G=G, \quad \text { and } \quad M G M=M .
$$

We have the following lemma. 
Lemma 3.5. [22, Theorem 2] Given a smooth matrix function $C: \mathbb{R} \rightarrow \mathbb{C}^{n, n}$, let $\lambda(t)$ be a simple eigenvalue of $C(t)$ and let $x(t)$ and $y(t)$ be the associated right and left eigenvectors, depending smoothly on $t$ and normalized such that $\|x(t)\|_{2}=\|y(t)\|_{2}=1$.

Moreover, let $M(t)=C(t)-\lambda(t) I$ and let $G(t)$ be the group inverse of $M(t)$. Then the eigenvectors satisfy the following system of differential equations:

$$
\begin{aligned}
\dot{x}(t) & =x(t) x(t)^{H} G(t) \dot{M}(t) x(t)-G(t) \dot{M}(t) x(t), \\
\dot{y}(t)^{H} & =y(t)^{H} \dot{M}(t) G(t) y(t) y(t)^{H}-y(t)^{H} \dot{M}(t) G(t) .
\end{aligned}
$$

In order to minimize the functional $F_{\varepsilon}(\Delta)$, we construct a family of matrices $A+\varepsilon \Delta(t)$, with $\Delta(t) \in \mathbb{C}^{n, n}$ and $\|\Delta(t)\|_{F}=1$, such that $\lim _{t \rightarrow \infty} \Delta(t)=\Delta_{\infty}$ and an eigenvector/eigenvalue pair $(x, \lambda)$ of $A+\varepsilon \Delta_{\infty}$ exists such that $F_{\varepsilon}(\Delta)$ is locally minimized (over all matrices $\Delta$ of unit norm).

If $F_{\varepsilon}(\Delta)>0$, then the derivative $\dot{\Delta}$ is chosen in the direction of steepest descent of $F_{\varepsilon}(\Delta)$ for the chosen eigenvalue/eigenvector pair of $A+\varepsilon \Delta$.

We start by considering the first summand $\frac{1}{2}|\lambda|^{2}$ in $F_{\varepsilon}(\Delta)$ (see (3.4)), which we assume to be different from zero.

Let $\lambda$ be a simple eigenvalue of $A+\varepsilon \Delta$ and let $x, y$ be the associated right and left eigenvectors, normalized according to (2.5). Proceeding as in (2.6) we get

$$
\frac{1}{2} \frac{d}{d t}|\lambda|^{2}=\varepsilon \alpha \operatorname{Re}\left\langle y x^{H}, \dot{\Delta}\right\rangle, \quad \text { with } \alpha=\frac{r}{\left|y^{H} x\right|},
$$

so that $\alpha$ is well-defined by the simplicity assumption and $\alpha \neq 0$ if $\lambda \neq 0$.

Next we consider the second summand $\frac{1}{2}\left\|V^{\mathrm{H}} x\right\|_{2}^{2}$ in $F_{\varepsilon}(\Delta)$, which we again assume to be different from zero. We get

$$
\frac{1}{2} \frac{d}{d t}\left\|V^{\mathrm{H}} x\right\|_{2}^{2}=\operatorname{Re}\left(x^{\mathrm{H}} V V^{\mathrm{H}} \dot{x}\right)=\nu \operatorname{Re}\left(u^{\mathrm{H}} \dot{x}\right)
$$

with

$$
u=V \frac{V^{\mathrm{H}} x}{\left\|V^{\mathrm{H}} x\right\|_{2}} \quad \text { and } \quad \nu=\left\|V^{H} x\right\|_{2} .
$$

This requires the derivative of the eigenvector $x$ of $A+\varepsilon \Delta(t)$, which is given by Lemma 3.5. Inserting (3.5) into (3.7) we obtain

$$
\begin{aligned}
\frac{1}{2} \frac{d}{d t}\left\|V^{\mathrm{H}} x\right\|_{2}^{2} & =\nu \operatorname{Re}\left(u^{\mathrm{H}} \dot{x}\right) \\
=\varepsilon \nu & \operatorname{Re}\left(u^{H}\left(\left(x^{H} G \dot{\Delta} x\right) x-G \dot{\Delta} x\right)\right) \\
& +\nu \operatorname{Re}\left(u^{H}\left(\left(x^{H} G \dot{\lambda} \mathrm{I} x\right) x-G \dot{\lambda} \mathrm{I} x\right)\right) .
\end{aligned}
$$

Using that for a simple eigenvalue $G x=0$ and $y^{H} G=0$, see [22], we get that the second summand of (3.8) vanishes. Hence

$$
\begin{aligned}
\frac{1}{2} \frac{d}{d t}\left\|V^{\mathrm{H}} x\right\|_{2}^{2} & =\varepsilon \nu^{2} \operatorname{Re}\langle x, G \dot{\Delta} x\rangle-\varepsilon \nu \operatorname{Re}\langle u, G \dot{\Delta} x\rangle \\
& =\varepsilon \nu \operatorname{Re}\left\langle G^{H}(\nu x-u) x^{H}, \dot{\Delta}\right\rangle=\varepsilon \nu \operatorname{Re}\left\langle v x^{H}, \dot{\Delta}\right\rangle,
\end{aligned}
$$


where $v:=G^{H}(\nu x-u)$. Therefore, with $w=\alpha y+\nu v$, we have

$$
\frac{d}{d t} F_{\varepsilon}(\Delta)=\varepsilon \operatorname{Re}\left\langle(\alpha y+\nu v) x^{H}, \dot{\Delta}\right\rangle=\varepsilon \operatorname{Re}\left\langle w x^{H}, \dot{\Delta}\right\rangle .
$$

Then, the optimal steepest descent direction $Z=\dot{\Delta}$ for $F_{\varepsilon}(\Delta)$ (see (3.6) and (3.8)) is determined by the following optimization problem, where $w$ is assumed to be different from zero.

$$
\begin{aligned}
Z_{*}=\arg \min _{Z \in \mathbb{C}^{n, n}} \operatorname{Re}\left\langle w x^{\mathrm{H}}, Z\right\rangle & \\
& \text { subject to } \operatorname{Re}\langle\Delta, Z\rangle=0 \text { and }\|Z\|_{F}=1 .
\end{aligned}
$$

The solution to the optimization problem (3.9) is given in the following lemma.

Lemma 3.6. Let $\Delta \in \mathbb{C}^{n, n}$ be of unit Frobenius norm, and let $w, x \in \mathbb{C}^{n} \backslash\{0\}$ be such that $\Delta$ is not proportional to $w x^{H}$. Then the solution of the optimization problem (3.9) is given by

$$
\kappa Z_{*}=-w x^{H}+\operatorname{Re}\left\langle\Delta, w x^{H}\right\rangle \Delta,
$$

where $\kappa$ is the Frobenius norm of the matrix on the right hand side.

Proof. The result follows by noting that the expression in (3.10) is the orthogonal projection of the rank-one matrix $w x^{H}$ to the subspace $\{Z: \operatorname{Re}\langle\Delta, Z\rangle=0\}$.

Lemma 3.6 suggests to consider the following gradient system for $F_{\varepsilon}(\Delta)$,

$$
\dot{\Delta}=-w x^{H}+\operatorname{Re}\left\langle\Delta, w x^{H}\right\rangle \Delta,
$$

where $y(t), x(t)$ are left and right eigenvectors, respectively, of unit norm associated with a simple eigenvalue $\lambda(t)=r \mathrm{e}^{\mathbf{i} \theta}$ of $A+\varepsilon \Delta(t)$ (for fixed $\varepsilon$ ), and with $y^{H} x=$ $\left|y^{H} x\right| \mathrm{e}^{-\mathbf{i} \theta}, w=\alpha y+\nu v, \alpha=|\lambda| /\left|y^{H} x\right|, v=G^{H}(\nu x-u), u=V V^{H} x / \nu$ and $\nu=$ $\left\|V^{H} x\right\|_{2}$.

The following result shows the monotonic decrease of $F_{\varepsilon}(\Delta(t))$ along every solution of (3.11).

THEOREM 3.7. Let $\Delta(t)$ of unit Frobenius norm satisfy the differential equation (3.11). If $\lambda(t)$ is a simple eigenvalue of $A+\varepsilon \Delta(t)$, then

$$
\frac{d}{d t} F_{\varepsilon}(\Delta(t)) \leq 0
$$

Proof. The result follows directly from Lemmas 2.1, 3.5 and 3.6.

The following lemma characterizes the right hand side of (3.11).

Lemma 3.8. Consider the functional (3.4) and suppose that $F_{\varepsilon}(\Delta)>0$. Let $\lambda$ be a simple eigenvalue of $A+\varepsilon \Delta$ (for fixed $\varepsilon$ ) and let $y, x$ be the associated left and right unit norm eigenvectors, respectively. If $v=G^{H}(\nu x-u)$, where $G$ is the group inverse of $A+\varepsilon \Delta-\lambda I$, then

$$
w=\alpha y+\nu v \neq 0 .
$$

Proof. (i) First assume that $\lambda \neq 0$. Exploiting the well-known property of the group inverse that $G x=0$ [22], we obtain that $x^{H} v=0$. 
If we had $\alpha y+\nu v=0$, then this would imply $x^{H}(\alpha y+\nu v)=0$ and thus, $\alpha x^{H} y=0$. Since $x^{H} y \neq 0$ by the simplicity of $\lambda$ and $\alpha \neq 0$ by the assumption $\lambda \neq 0$, it follows that $\left|\alpha x^{H} y\right|=|\lambda|$, which is a contradiction.

(ii) Next assume that $\lambda=0$. This implies $\alpha=0$ and $\nu \neq 0$. Then $w=\nu v$ with $v=\frac{1}{\nu}\left(\nu^{2} x-V V^{H} x\right)$. Since $\lambda$ is simple, the group inverse $G$ of $A+\varepsilon \Delta-\lambda \mathrm{I}$ has rank $n-1$. This implies that $\operatorname{ker}\left(G^{H}\right)=\operatorname{span}(y)$, and hence $w=0$. Therefore

$$
\nu^{2} x-V V^{H} x=\eta y
$$

for some $\eta \in \mathbb{C}, \eta \neq 0$. As a consequence

$$
0=x^{\mathrm{H}}\left(\nu^{2} x-V V^{H} x\right)=\eta x^{H} y
$$

which gives a contradiction, since $x^{H} y \neq 0$.

Lemma 3.8 implies that at a stationary point of (3.11) for which $F_{\varepsilon}(\Delta)>0$, the summands in the right-hand side of (3.11) cannot vanish simultaneously.

Stationary points of (3.11), which are potential minimizers for the computation of $F_{\varepsilon}(\Delta)$, can be characterized as follows.

THEOREM 3.9. Consider the functional (3.4) and suppose that $F_{\varepsilon}(\Delta)>0$. Let $\lambda$ be a simple eigenvalue of $A+\varepsilon \Delta$ (for fixed $\varepsilon$ ) and let $y, x$ be the associated left and right unit norm eigenvectors, respectively. Then the following are equivalent on solutions of (3.11).

(1) $\frac{d}{d t} F_{\varepsilon}(\Delta)=0$;

(2) $\dot{\Delta}=0$;

(3) $\Delta$ is a real multiple of $w x^{H}$ (where $w=\alpha y+\nu v$ ).

Proof. The proof follows directly by equating to zero the right hand side of (3.11) and by Lemma 3.8, which prevents that $w=0$.

The following theorem characterizes the local minimizers.

THEOREM 3.10. Consider the functional (3.4) and suppose that $F_{\varepsilon}(\Delta)>0$. Let $\Delta_{*} \in \mathbb{C}^{n, n}$ with $\left\|\Delta_{*}\right\|_{F}=1$. Let $\lambda_{*}=r \mathrm{e}^{\mathrm{i} \theta}$ be a simple eigenvalue of $A+\varepsilon \Delta_{*}$ with left and right eigenvectors $y$ and $x$, respectively, both of unit norm and with the normalization $y^{H} x=\left|y^{H} x\right| \mathrm{e}^{-\mathbf{i} \theta}$ and $F_{\varepsilon}(\Delta)>0$. Then the following are equivalent:

(i) Every differentiable path $\left(\Delta(t), \lambda(t)\right.$ ) (for small $t \geq 0$ ) such that $\|\Delta(t)\|_{F} \leq 1$ and $\lambda(t)$ is a simple eigenvalue of $A+\varepsilon \Delta(t)$, with $\Delta(0)=\Delta_{*}$, satisfies

$$
\frac{d}{d t} F_{\varepsilon}(\Delta(t)) \geq 0
$$

(ii) $\Delta_{*}$ is a negative multiple of $w x^{H}$, where $w=\alpha y+\nu v$.

Proof. First of all note that Lemma 3.8 ensures that $w x^{H} \neq 0$.

Assume that (i) does not hold. Then there exists a path $\Delta(t)$ through $\Delta_{*}$ such that $\left.\frac{d}{d t} F(\Delta(t))\right|_{t=0}<0$. The minimization property established by Lemma 3.6 together with Lemmas 2.1, and 3.5 shows that also the solution path of (3.11) passing through $\Delta *$ is such a path. Hence $\Delta_{*}$ is not a stationary point of $(3.11)$, and Theorem 3.9 then yields that $\Delta_{*}$ is not a real multiple of $w x^{H}$. This implies that also (ii) does not hold.

Conversely, if $\Delta_{*}$ is not a real multiple of $w x^{H}$, then $\Delta_{*}$ is not a stationary point of (3.11), and Theorems 3.9 and 3.7 yield that $\left.\frac{d}{d t} F(\Delta(t))\right|_{t=0}<0$ along the solution path of (3.11). 
Moreover, using a similar argument to [9, Theorem 2.2], if

$$
\Delta_{*}=\gamma w x^{H}, \quad \text { with } \quad \gamma>0,
$$

then along the path $\Delta(t)=(1-t) \Delta_{*}, t \in[0, \tau](\tau>0)$, which is such that $\|\Delta(t)\|_{F} \leq 1$ if $\tau \leq 2$, we have that

$$
\frac{d}{d t} F(\Delta(t))=-\gamma\left\|w x^{H}\right\|_{F}^{2}<0
$$

Hence, by exploiting Lemmas 2.1 and 3.5 , as well as $\left.\frac{d}{d t} F(\Delta(t))\right|_{t=0}<0$, this contra$\operatorname{dicts}(\mathrm{i})$.

As a consequence, if in Theorem 3.9 we have that $F_{\varepsilon}(\Delta)>0$ is locally minimal, then

$$
\Delta=-\frac{w}{\|w\|_{F}} x^{H}
$$

which is a rank-one matrix.

3.3. Rank-one property of the solution of the common null vector problem with fixed $E$. In [21] it was conjectured that the minimal norm perturbation that makes a pencil $(A, E)$ singular, when only $A$ is perturbed, is of rank one. While this does not appear to be true in general (as is indicated by Example 2.10), it does hold for the restricted case of the common nullspace problem.

Theorem 3.11. Consider a regular pencil $(A, E)$. Then the perturbation $\Delta A$ of minimal Frobenius norm such that $A+\Delta A$ and $E$ have a common null vector, is such that $\Delta A$ has rank one.

Proof. The result is a consequence of the rank-one property of extremizers. In fact, we have shown above that for any $\varepsilon$ such that $F_{\varepsilon}(\Delta(\varepsilon))>0$ for $\varepsilon<\varepsilon^{\star}$ and $F_{\varepsilon}\left(\Delta\left(\varepsilon^{\star}\right)\right)=0$, the extremizers $\Delta(\varepsilon)$ of $F_{\varepsilon}(\Delta)$ have rank one. They converge to a rank-one matrix as $\varepsilon \rightarrow \varepsilon^{\star}$ by the lower semi-continuity of the rank.

Since for a rank-one matrix the Frobenius norm and the matrix 2-norm are the same, Theorem 3.11 further shows that there is a perturbation $\Delta A$ of minimal 2-norm such that $A+\Delta A$ and $E$ have a common null vector, which has rank one. There may, however, be further perturbations of the same 2-norm that have arbitrary rank.

3.4. Rank-one dynamics to compute the distance to singularity with common null space. Since the extremizers of (3.4) are of rank one, we can proceed in complete analogy to [8] to obtain a suitable ODE on the manifold $\mathcal{M}_{1}$ of rank-one matrices in $\mathbb{C}^{n, n}$.

We express $\Delta \in \mathcal{M}_{1}$ as $\Delta=\sigma p q^{H}$, where $\sigma \in \mathbb{C}, p, q \in \mathbb{C}^{n}$ have unit norm, and the derivatives $\dot{\sigma} \in \mathbb{C}, \dot{p}, \dot{q} \in \mathbb{C}^{n}$ will be uniquely determined from $\sigma, p, q$ and $\dot{\Delta}$ by imposing the orthogonality conditions $p^{H} \dot{p}=0, q^{H} \dot{q}=0$.

In the differential equation (3.11) we replace the right-hand side by its orthogonal projection to the tangent space $T_{\Delta} \mathcal{M}_{1}$ of the manifold $\mathcal{M}_{1}$ and obtain

$$
\dot{\Delta}=P_{\Delta}\left(-w x^{H}+\operatorname{Re}\left\langle\Delta, w x^{H}\right\rangle \Delta\right),
$$

where $x$ and $y$ are again unit norm right and left eigenvectors, respectively, associated with a simple eigenvalue $\lambda$ of $A+\varepsilon \Delta$, with $y^{H} x>0$ and $w=\alpha y+\nu v$, where $\alpha, \nu$ and $v$ are defined as before. 
The orthogonal projection onto $T_{\Delta} \mathcal{M}_{1}$ at $\Delta=\sigma p q^{H} \in \mathcal{M}_{1}$ is given by

$$
P_{\Delta}(Z)=Z-\left(\mathrm{I}-p p^{H}\right) Z\left(\mathrm{I}-q q^{H}\right)
$$

for some $Z \in \mathbb{C}^{n, n}$. With this formula we immediately obtain the following lemma.

Lemma 3.12. Consider the orthogonal projection $P_{\Delta}(Z)$ as in (3.15). For $\Delta=$ $\sigma p q^{H} \in \mathcal{M}_{1}$ with nonzero $\sigma \in \mathbb{C}$ and with $p \in \mathbb{C}^{n}$ and $q \in \mathbb{C}^{n}$ of unit norm, the equation $\dot{\Delta}=P_{\Delta}(Z)$ is equivalent to $\dot{\Delta}=\dot{\sigma} p q^{H}+\sigma \dot{p} q^{H}+\sigma p \dot{q}^{H}$, where

$$
\begin{aligned}
\dot{\sigma} & =p^{H} Z q, \\
\dot{p} & =\left(I-p p^{H}\right) Z q \sigma^{-1}, \\
\dot{q} & =\left(I-q q^{H}\right) Z^{H} p \bar{\sigma}^{-1} .
\end{aligned}
$$

Proof. The proof follows by using the rank-one representation of $\Delta$; see [8].

Inserting $Z=-w x^{H}+\operatorname{Re}\left\langle\Delta, w x^{H}\right\rangle \Delta$, we get that the differential equation (3.14) for $\Delta=\sigma p q^{H}$ can be rewritten as the following system of differential equations for $\sigma, p$ and $q$. Here we set $\eta=-p^{H} w \in \mathbb{C}, \beta=q^{H} x \in \mathbb{C}$.

$$
\begin{aligned}
\dot{\sigma} & =\eta \bar{\beta}-\operatorname{Re}(\bar{\eta} \beta \sigma) \sigma=\mathbf{i} \operatorname{Im}(\eta \bar{\beta} \bar{\sigma}) \sigma, \\
\dot{p} & =(-w-\eta p) \bar{\beta} \sigma^{-1}, \\
\dot{q} & =(x-\beta q) \bar{\eta} \bar{\sigma}^{-1} .
\end{aligned}
$$

The derivation of this system of ODEs is straightforward; see [9] for the details.

A nice consequence of this reformulation of the gradient system is that the monotonicity and the characterization of stationary points is analogous to those obtained for (3.11), i.e., we have Theorems 3.7 and 3.9 (for the proofs we refer to [8]). This means that the rank-one ODE has the same stationary points as (3.11), which include the desired extremizers.

As a consequence we can integrate the ODE (3.17) instead of (3.11) in the inner iteration of the optimization procedure. Based on this, we present in the next subsection a corresponding numerical method for the outer iteration.

3.5. The outer iteration, updating $\varepsilon$. In this subsection we discuss the outer iteration to update $\varepsilon$. For every $\varepsilon>0$, the gradient system (3.11) yields a stationary point $\Delta(\varepsilon)$ of unit Frobenius norm that is a (local) minimum of $F_{\varepsilon}$. The method is constructed to approach, from the left-hand side, the value $\varepsilon^{\star}>0$ such that $F_{\varepsilon^{\star}}\left(\Delta\left(\varepsilon^{\star}\right)\right)=0$ and $F_{\varepsilon}(\Delta(\varepsilon))>0$ for $\varepsilon<\varepsilon^{\star}$. We make the following generic assumption.

Assumption 3.13. The smallest eigenvalue $\lambda(\varepsilon)$ of the extremal matrix $A+\varepsilon \Delta(\varepsilon)$ is simple. Moreover, we assume that $\Delta(\varepsilon)$ and $\lambda(\varepsilon), x(\varepsilon)$ are smooth with respect to $\varepsilon$ (at least in a neighborhood of $\varepsilon^{\star}$ ).

The following result provides an explicit and easily computable expression for the derivative of $F_{\varepsilon}(\Delta(\varepsilon))$ with respect to $\varepsilon$.

THEOREM 3.14. Consider the optimization problem of determining a rank-one perturbation minimizing $F_{\varepsilon}(\Delta(\varepsilon))$ by perturbing only A. Suppose that:

1. $\varepsilon \in\left(0, \varepsilon^{\star}\right)$ such that $F_{\varepsilon}(\Delta(\varepsilon))>0$,

2. Assumption 3.13 holds.

Then,

$$
\frac{d F_{\varepsilon}(\Delta(\varepsilon))}{d \varepsilon}=-\left\|w(\varepsilon) x(\varepsilon)^{H}\right\|_{F}<0, \quad \text { for all } \varepsilon
$$


where

$w(\varepsilon)=\alpha(\varepsilon) y(\varepsilon)+\nu(\varepsilon) v(\varepsilon), \quad \alpha(\varepsilon)=\frac{|\lambda(\varepsilon)|}{\left|y(\varepsilon)^{H} x(\varepsilon)\right|}, \quad v(\varepsilon)=G(\varepsilon)^{H}(\nu(\varepsilon) x(\varepsilon)-u(\varepsilon))$,

and

$$
u(\varepsilon)=V \frac{V^{H} x(\varepsilon)}{\left\|V^{H} x(\varepsilon)\right\|}, \quad \nu(\varepsilon)=\left\|V^{\mathrm{H}} x(\varepsilon)\right\|_{2}
$$

Proof. The proof is similar to that of Theorem 2.9.

Having obtained a computational method to compute the minimal rank-one perturbation of $A$ which makes the pencil $(A, E)$ singular, we prove below that actually this gives the solution to the general rank perturbation.

3.6. Numerical examples for the common nullspace problem with fixed $E$. In this subsection we present several illustrative numerical examples.

EXAMPLE 3.15. Consider the pencil from Example 2.11. Running the algorithm presented in the last subsection to the pair $\left(A^{H}, E^{H}\right)$ we obtain the same distance $\varepsilon^{\star} \approx 1.8907$ as computed by means of the general algorithm, with the rank-one perturbation to be added to $A$ given by $\Delta=\bar{\sigma} q p^{H}$ with

$$
\begin{aligned}
\sigma & =0.9987-0.0506 \mathbf{i}, \\
p & =\left[\begin{array}{lll}
-0.8277-0.4597 \mathbf{i} & 0.0462+0.0257 \mathbf{i} & -0.2775-0.1541 \mathbf{i}
\end{array}\right]^{T}, \\
q & =\left[\begin{array}{lll}
-0.8975-0.4406 \mathbf{i} & -0.0066-0.0010 \mathbf{i} & 0.0132+0.0117 \mathbf{i}
\end{array}\right] .
\end{aligned}
$$

EXAmple 3.16. Consider the matrices

$$
A=\left[\begin{array}{rrrrrr}
0.49 & 0.89 & 0.33 & 0.32 & 1.09 & -0.01 \\
1.03 & -1.15 & -0.75 & 0.31 & 1.11 & 1.53 \\
0.73 & -1.07 & 1.37 & -0.86 & -0.86 & -0.77 \\
-0.30 & -0.81 & -1.71 & -0.03 & 0.08 & 0.37 \\
0.29 & -2.94 & -0.10 & -0.16 & -1.21 & -0.23 \\
-0.79 & 1.44 & -0.24 & 0.63 & -1.11 & 1.12
\end{array}\right], E=\left[\begin{array}{llllll}
0 & 0 & 0 & 0 & 1 & 0 \\
0 & 1 & 0 & 1 & 0 & 0 \\
0 & 1 & 0 & 1 & 0 & 0 \\
1 & 0 & 0 & 0 & 1 & 1 \\
1 & 1 & 0 & 0 & 0 & 1 \\
1 & 0 & 0 & 0 & 0 & 1
\end{array}\right]
$$

The matrix $E$ has rank 4 and an orthonormal basis for $\operatorname{ker}(E)^{\perp}$ is given by

$$
V=\left[\begin{array}{rrrr}
0.6196 & -0.2399 & -0.1986 & -0.1384 \\
0.3846 & 0.6684 & -0.0501 & 0.6347 \\
0.0000 & 0.0000 & 0.0000 & -0.0000 \\
0.1532 & 0.6229 & 0.2343 & -0.7304 \\
0.2469 & -0.2236 & 0.9294 & 0.1592 \\
0.6196 & -0.2399 & -0.1986 & -0.1384
\end{array}\right]
$$

The simple bounds

$$
0.1936 \leq d_{A}^{0}(A, E) \leq 7.2810
$$

are computed according to the procedure given in Subsection 3.1. 
Applying the ODE-based approach, we obtain $\varepsilon^{\star} \approx 1.21$ and the corresponding matrix $\Delta$ is given by

$$
\Delta_{*}=p x^{H}, \quad x \approx\left[\begin{array}{r}
-0.574 \\
0.000 \\
0.583 \\
0.000 \\
0.000 \\
0.574
\end{array}\right], \quad p \approx\left[\begin{array}{r}
-0.075+0.134 \mathbf{i} \\
-0.125-0.029 \mathbf{i} \\
-0.052+0.065 \mathbf{i} \\
-0.498+0.096 \mathbf{i} \\
-0.295+0.021 \mathbf{i} \\
0.779+0.041 \mathbf{i}
\end{array}\right] .
$$

The monotonicity of the functional $F_{\varepsilon}$ in $\varepsilon=\varepsilon^{\star}$ is shown in Figure 3.1.

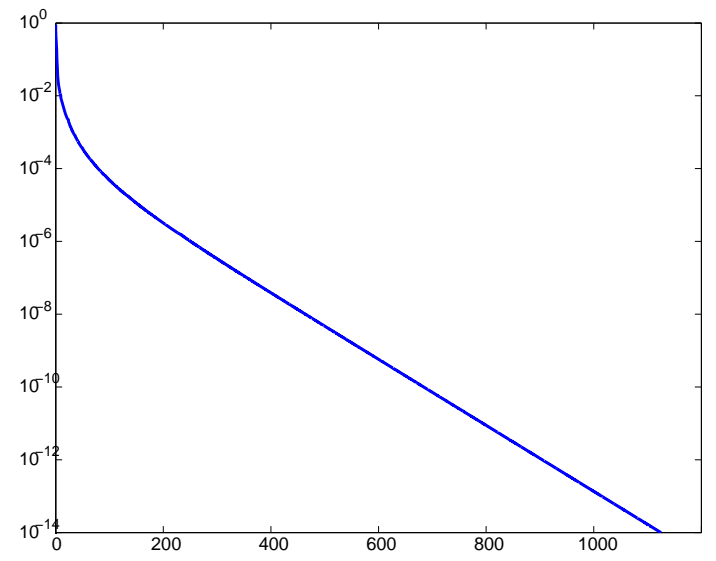

FIG. 3.1. The function $t \rightarrow F_{\varepsilon^{\star}}(\Delta(t))$ for Example 3.16.

EXAmple 3.17. Consider, see [6],

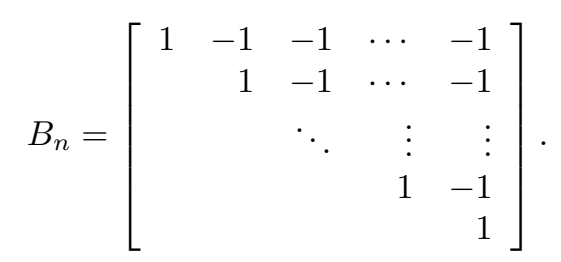

This is an ill-conditioned triangular matrix with no small diagonal entry. The rankone perturbation $\Delta_{B}=-2^{2-n} e_{n} e_{1}^{T}$ makes $B_{n}$ singular.

Thus for the pencil $(A, E)=\left(B_{n}, B_{n}+\Delta_{B}\right)$ if we perturb $A$ by $\Delta_{B}$ leads to the distance to singularity $d_{A}^{0}(A, E) \leq 2^{2-n}$.

In the general case the null vector of $E$ is

$$
x=\left[\begin{array}{llllll}
2^{n-2} & 2^{n-1} & \ldots & 2 & 1 & 1
\end{array}\right]^{\mathrm{T}}
$$

and we can look for

$$
\Delta A=\left[\begin{array}{rrrrr}
0 & 0 & \ldots & 0 & 0 \\
0 & 0 & \ldots & 0 & 0 \\
0 & 0 & \ldots & 0 & 0 \\
\delta_{n} & \delta_{n-1} & \ldots & \delta_{2} & \delta_{1}
\end{array}\right]
$$

21 
such that $\left(\delta+e_{1}\right)^{\mathrm{T}} x=0$, where

$$
\delta=\left[\begin{array}{llllll}
\delta_{n} & \delta_{n-1} & \ldots & \delta_{3} & \delta_{2} & \delta_{1}
\end{array}\right]^{\mathrm{T}}
$$

and $\|\delta\|_{F}$ is minimal. The minimization problem

$$
\min _{\delta_{1}, \ldots, \delta_{n}} \sum_{i=1}^{n} \delta_{i}^{2} \quad \text { s.t. } \quad \sum_{i=1}^{n} \delta_{i} x_{i}=-1
$$

is easily solvable and the solution is

$$
\delta=-x /\|x\|^{2} \quad \text { such that } \quad\|\Delta A\|_{F}=\frac{1}{\|x\|} .
$$

Note that imposing a left common null vector would lead to the same distance and the optimal perturbation would have the first column equal to the last row of $\Delta A$ in reverse order.

Consider for example the case $n=5$, where $d_{A}^{0}(A, E) \leq 1 / 8=0.125$. The exact closest singular pair is obtained with the perturbation

$$
\Delta A=-\left[\begin{array}{ccccc}
0 & 0 & 0 & 0 & 0 \\
0 & 0 & 0 & 0 & 0 \\
0 & 0 & 0 & 0 & 0 \\
0 & 0 & 0 & 0 & 0 \\
\frac{4}{43} & \frac{2}{43} & \frac{1}{43} & \frac{1}{22} & \frac{1}{86}
\end{array}\right]
$$

having (up to four digits) the Frobenius norm $\|\Delta A\|_{F}=\frac{1}{\sqrt{86}}=0.107833$.

The ODE method correctly finds an accurate approximation of $\Delta A$ also for larger values of $n$. For example, for $n=10$, it correctly approximates the distance and the optimal perturbation to six digits.

Finally, according to our experiments, $d_{A}(A, E)<d_{A}^{0}(A, E)$. For example, for $n=10$, we obtain $d(A, E) \approx 0.0030, d_{A}(A, E) \approx 0.030$ and $d_{A}^{0}(A, E) \approx 0.034$.

3.7. Numerical integration of the gradient system. For the numerical integration of the gradient system (3.17) we apply a suitable variable stepsize projected Euler method, in order to preserve the norm of $p$ and $q$ and the modulus 1 property of $\sigma$. In order to control the stepsize, we simply require the monotonicity property of the exact flow, i.e., $F\left(\Delta\left(t_{\ell+1}\right)\right)<F\left(\Delta\left(t_{\ell}\right)\right)$. For this reason we do not estimate $\left\|\Delta\left(t_{\ell+1}\right)-\Delta_{\ell+1}\right\|$ as we do not make use of any classical error estimate on the solution.

Concerning the outer iteration, by definition, the function $F_{\varepsilon}(\Delta(\varepsilon))$ has generically a double zero at $\varepsilon=\varepsilon^{\star}$ and then vanishes identically for $\varepsilon>\varepsilon^{\star}$. This suggests to approach the root from the left, while values $\varepsilon>\varepsilon^{\star}$ may only provide upper bounds.

Let $f(\varepsilon)=F_{\varepsilon}(\Delta(\varepsilon))$. Since for $\varepsilon<\varepsilon^{\star}$ we can exploit the knowledge of $f(\varepsilon)$ as well as $f^{\prime}(\varepsilon)$. Similarly to the case discussed in Section 2.2, for $\varepsilon_{k}<\varepsilon^{\star}$ we can set

$$
\varepsilon_{k+1}=\varepsilon_{k}-2 \frac{f\left(\varepsilon_{k}\right)}{f^{\prime}\left(\varepsilon_{k}\right)}
$$

and obtain a quadratically convergent iteration from the left.

At every step of the outer iteration we need to compute the smallest eigenvalue and the associated eigenvectors of the matrix $A+\varepsilon \Delta_{n}$. For small dense problems we use the $Q R$ algorithm implemented in eig, while for problems of large dimension (and possibly 
sparse structure) we make use of the routine eigs, which is based on ARPACK [19] and implements the implicitly restarted Arnoldi method. A major computational problem when computing the right-hand side of the differential equations (3.11) and (3.17) is the the application of the group inverse $G$ to a vector. In order to compute it efficiently we make use of the following result from [11].

THEOREM 3.18. Suppose that the matrix $B$ has a semisimple eigenvalue 0 with $x \in \operatorname{ker}(B)$ and $y \in \operatorname{ker}\left(B^{H}\right)$ of unit norm and such that $y^{H} x>0$. Let $G$ be the group inverse of $B$. Then

$$
G=\Pi B^{\dagger} \Pi=\Pi\left(B+\eta y x^{H}\right)^{-1} \Pi,
$$

where $\eta \in \mathbb{R}, B^{\dagger}$ is the Moore-Penrose pseudoinverse of $B, \Pi$ is the projection $\left(I-\eta x \hat{y}^{H}\right)$, with $\hat{y}=y /\left(y^{H} x\right)$ and $\eta \neq 0$ is arbitrary.

In our case we apply Theorem 3.18 with $B=A-\lambda I+\varepsilon \Delta$, so that $G=$ $\Pi(A-\lambda I+\varepsilon \Gamma)^{-1} \Pi$, where

$$
\Gamma=g x^{H}, \quad g=-w+\hat{y}
$$

is a rank-one matrix. Hence we can make use of the Sherman-Morrison-Woodbury formula, see [6], so that with $L=(A-\lambda I)^{-1}$, we obtain

$$
(A-\lambda I+\varepsilon \Gamma)^{-1}=L-\frac{1}{\left(1+\varepsilon x^{H} L \gamma\right)} L \gamma x^{H} L .
$$

This allows for an efficient computation of the application of $G$ to a vector. Note also that $G$ is well-conditioned if $\lambda$ is not close to another eigenvalue.

We usually observe that the term $|\lambda(\Delta)|^{2}$ is more rapidly converging to zero than the term $\left\|V^{H} x(\Delta)\right\|_{2}^{2}$. This leads to an oscillating behavior close to zero of the first term, which causes a stepsize restriction and a consequent slowing down of the convergence close to the stationary point, see Figure 3.2.

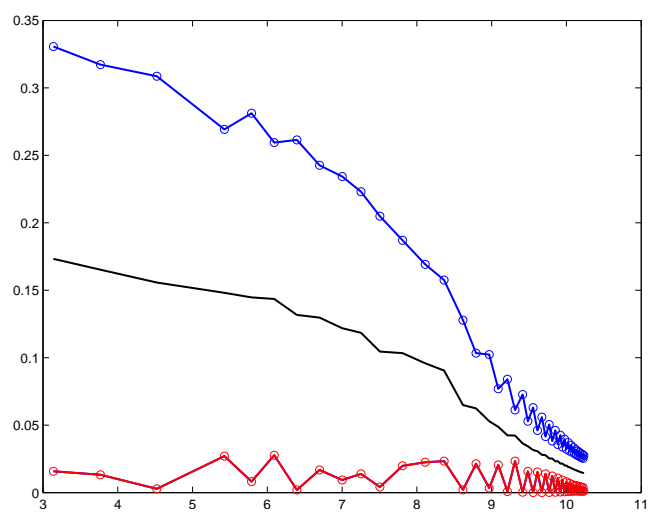

FIG. 3.2. Zoom of the two terms $|\lambda|^{2}$ (in red) and $\left\|V^{H} x\right\|_{2}^{2}$ (in blue) and the functional $F$ (in black) versus time for the illustrative example of Section 3.6 (with $\varepsilon=1$ )

In this section we have obtained convincing results and a quadratically convergent method to compute the distance to a singular pencil with common null vector, where only one of the matrices is perturbed. In the next section we consider again the common null vector problem but allow perturbations in both matrices. 
4. Distance to singular pencils with common null vectors. We now study the distance to a pencil with common null vector when both matrices $A$ and $E$ are perturbed.

4.1. An ODE-based approach for computing $d^{0}(A, E)$. We look for the closest pair of matrices $(A+\Delta A, E+\Delta E)$ so that $A+\Delta A$ is singular with an eigenvector $x$ to the eigenvalue zero belonging to $\operatorname{ker}(E+\Delta E)$. In this way we would get

$$
(A+\Delta A+\mu(E+\Delta E)) x=0 \quad \text { for all } \mu \in \mathbb{C} .
$$

This problem can be reformulated as

$$
\begin{aligned}
\left(\Delta A_{*}, \Delta E_{*}\right)=\arg & \min _{\Delta A, \Delta E \in \mathbb{C}^{n, n}}\|(\Delta A, \Delta E)\| \\
& \text { subject to }(A+\Delta A) x=0 \\
& \text { and }(E+\Delta E) x=0 \text { for some } x \in \mathbb{C}^{n}, x \neq 0 .
\end{aligned}
$$

We then have $d^{0}(A, E)=\left\|\left(\Delta A_{*}, \Delta E_{*}\right)\right\|$.

In the inner iteration for the perturbation size $\varepsilon$ we consider the perturbed matrices $A+\varepsilon \Delta$ and $E+\varepsilon \Theta$ with $\|(\Delta, \Theta)\|_{F}=1$. Let us denote by $(\lambda, x, y)$ an eigentriple of $A+\varepsilon \Delta$ (with $\lambda$ to be driven to zero) and by $(\nu, a, b)$ an eigentriple of $E+\varepsilon \Theta$ (with $\nu$ to be driven to zero), where the eigenvectors are scaled according to (2.5).

The functional we aim to minimize in order to compute the closest pair with a common right null vector is given by

$$
F_{\varepsilon}(\Delta, \Theta)=\frac{1}{2}\left(\|\lambda\|_{2}^{2}+\|\nu\|_{2}^{2}+\left(1-\left|x^{H} a\right|^{2}\right)\right)
$$

This is phase-invariant with respect to the eigenvectors $x$ and $a$, which we assume to be normalized such that $\|x\|=\|a\|=1$.

Similarly, for computing the closest pair with a common left null vector, we simply replace $(A, E)$ by $\left(A^{H}, E^{H}\right), x$ by $y$ and $a$ by $b$. The computation of the gradient is obtained similarly to previous cases, and we obtain the expressions

$$
\begin{aligned}
& \frac{1}{2} \frac{d}{d t}|\lambda|^{2}=\alpha \varepsilon \operatorname{Re}\left\langle y x^{H}, \dot{\Delta}\right\rangle, \quad \text { with } \alpha=\frac{|\lambda|}{\left|y^{H} x\right|}, \\
& \frac{1}{2} \frac{d}{d t}|\nu|^{2}=\beta \varepsilon \operatorname{Re}\left\langle b a^{H}, \dot{\Theta}\right\rangle, \quad \text { with } \beta=\frac{|\nu|}{\left|b^{H} a\right|}
\end{aligned}
$$

as before. The other term instead, is given by

$$
\left.\frac{1}{2} \frac{d}{d t}\left(\left|x^{H} a\right|^{2}\right)=\frac{1}{2} \frac{d}{d t}\left(x^{H} a a^{H} x\right)=\operatorname{Re}\left(x^{H} a a^{H} \dot{x}\right)+\operatorname{Re}\left(a^{H} x x^{H} \dot{a}\right)\right) .
$$

This yields

$$
\begin{aligned}
& \frac{1}{2} \frac{d}{d t}\left(\left|x^{H} a\right|^{2}\right)= \varepsilon \operatorname{Re}\left(\left|x^{H} a\right|^{2} x^{H} G \dot{\Delta} x-\left(x^{H} a\right) a^{H} G \dot{\Delta} x\right) \\
&+ \varepsilon \operatorname{Re}\left(\left|x^{H} a\right|^{2} a^{H} N \dot{\Theta} a-\left(a^{H} x\right) x^{H} N \dot{\Theta} a\right) \\
&=\varepsilon \operatorname{Re}\left(\left\langle|\theta|^{2} G^{H} x x^{H}-\bar{\theta} G^{H} a x^{H}, \dot{\Delta}\right\rangle\right. \\
&\left.+\left\langle|\theta|^{2} N^{H} a a^{H}-\theta N^{H} x a^{H}, \dot{\Theta}\right\rangle\right)
\end{aligned}
$$


where $\theta=x^{H} a, G$ is the group inverse of $A+\varepsilon \Delta-\lambda I$, and $N$ is the group inverse of $E+\varepsilon \Theta-\nu I$.

In order to minimize the gradient of $F$ we collect the summands involving $\dot{\Delta}$ and those involving $\dot{\Theta}$. We get

$$
\frac{d}{d t} F_{\varepsilon}(\Delta, \Theta)=\varepsilon \operatorname{Re}\left\langle(\alpha y-v) x^{H}, \dot{\Delta}\right\rangle+\varepsilon \operatorname{Re}\left\langle(\beta b-w) a^{H}, \dot{\Theta}\right\rangle,
$$

with $v=|\theta|^{2} G^{H} x-\bar{\theta} G^{H} a$ and $w=|\theta|^{2} N^{H} a-\theta N^{H} x$.

This leads to the system of ODEs,

$$
\begin{aligned}
& \dot{\Delta}=-(\alpha y-v) x^{H}+\eta \Delta, \\
& \dot{\Theta}=-(\beta b-w) a^{H}+\eta \Theta,
\end{aligned}
$$

where

$$
\eta=\operatorname{Re}\left\langle\Delta,(\alpha y-v) x^{H}\right\rangle+\operatorname{Re}\left\langle\Theta,(\beta b-w) a^{H}\right\rangle
$$

ensures the norm conservation.

We obtain similar results to those given for the case of fixed $E$. In particular we have the following result which characterizes the right hand side of (4.4).

LEMMA 4.1. Let $F_{\varepsilon}(\Delta, \Theta)>0$; let $\lambda$ be a simple eigenvalue of $A+\varepsilon \Delta$ ( $\varepsilon$ is fixed) and let $y, x$ be the left and right associate eigenvectors scaled according to (2.5). Let $\nu$ be a simple eigenvalue of $E+\varepsilon \Theta$ and let $b, a$ be the left and right associate eigenvectors scaled according to (2.5). If $\theta=x^{\mathrm{H}} a \neq 0$, then

$$
\alpha y-v \neq 0 \quad \text { and } \quad \beta b-w \neq 0
$$

Proof. We prove that $u_{1}=\alpha y-v$ cannot vanish, the proof for $u_{2}=\beta b-w$ uses the same arguments. (i) First assume that $\lambda \neq 0$. Exploiting the property $G x=0$, [22], we obtain that $x^{H} v=0$. Then, assume by contradiction that $u_{1}=0$. This would imply $x^{H}(\alpha y-v)=0$ and hence $\alpha x^{H} y=0$. Since $x^{H} y \neq 0$ by the simplicity of $\lambda$, and since $\alpha \neq 0$ by the assumption $\lambda \neq 0$, it follows that $\left|\alpha x^{H} y\right|=|\lambda|$, which gives a contradiction.

(ii) If $\lambda=0$ then $\alpha=0$ and $u_{1}=v$ with $v=|\theta|^{2} G^{H} x-\bar{\theta} G^{H} a$. By the simplicity assumption on $\lambda$, the group inverse $G$ of $A+\varepsilon \Delta$ has rank $n-1$. This implies that $\operatorname{ker}\left(G^{H}\right)=\operatorname{Span}(y)$. Hence, $u_{1}=0$ implies that $|\theta|^{2} x-\bar{\theta} a=\eta y$ for some $\eta \in \mathbb{C}, \eta \neq 0$. As a consequence $0=x^{\mathrm{H}}\left(|\theta|^{2} x-\bar{\theta} a\right)=\eta x^{H} y$ which is a contradiction, since $x^{H} y \neq 0$. ㄱ

4.2. Rank-one property of the common nullspace problem. In the same way as in Section 3.3, we obtain from (4.4) and Lemma 4.1 the following property.

THEOREM 4.2. Consider a regular pencil $(A, E)$. Then the perturbation of minimal Frobenius norm $(\Delta A, \Delta E)$ such that $A+\Delta A$ and $E+\Delta E$ have a common null vector, is such that $\Delta A$ and $\Delta E$ each have rank at most one.

4.3. Numerical examples. We consider here the same matrices of Section 3.6.

Consider first Example 2.11. Applying the ODE method leads to the bound (to seven digits) $d^{0}(A, E) \leq 0.9438619$, which is about the half of $d_{A}^{0}(A, E)$. The closest 
computed singular pencil (with a left common null vector) is

$$
\begin{aligned}
& A+\varepsilon \Delta \approx {\left[\begin{array}{rrr}
-1.6212384085 & 0.3554202002 & -0.5130886135 \\
1.0469394294 & -0.2266297260 & 0.5965969015 \\
-0.9441526182 & 0.2179904923 & 0.7121049288
\end{array}\right], } \\
& E+\varepsilon \Theta \approx\left[\begin{array}{rrr}
-0.0000071285 & 0.1271034578 & -0.4844321165 \\
0.0000052330 & 0.1505583462 & 0.4262604190 \\
-0.0000023335 & 0.9605965130 & 0.1501681259
\end{array}\right] .
\end{aligned}
$$

Note that the first column of $E+\varepsilon \Theta$, which should be 0 analytically, vanishes within the stopping tolerance of the method $\left(10^{-6}\right)$. The rank-one matrices $\Delta$ and $\Theta$ have norms: $\|\Delta\|_{F} \approx 0.544470$ and $\|\Theta\|_{F} \approx 0.838781$.

Consider next Example 3.17 in Section 3.6. Applying the ODE method for the case $n=5$ leads to the bound (up to seven digits) $d^{0}(A, E) \leq 0.100177$, which is slightly smaller than $d_{A}^{0}(A, E)=0.107833$. The associated computed nearby singular pencil (having a common right null vector) is

$$
\begin{aligned}
& A+\varepsilon \Delta \approx \\
& {\left[\begin{array}{rrrrr}
0.9976840743 & -1.0009737845 & -1.0004216057 & -1.0002259729 & -0.9997389967 \\
-0.0043828536 & 0.9981784774 & -1.0007637414 & -1.0003813706 & -0.9996427155 \\
-0.0090482003 & -0.0038535312 & 0.9983442615 & -1.0008191138 & -0.9995477484 \\
-0.0192177591 & -0.0083685364 & -0.0036814287 & 0.9981783278 & -0.9994870221 \\
-0.0832976242 & -0.0387575470 & -0.0183979414 & -0.0095660488 & 0.9986398210
\end{array}\right],} \\
& E+\varepsilon \Theta \approx \\
& {\left[\begin{array}{rrrrr}
0.9978049925 & -1.0010539505 & -1.0005015794 & -1.0002252097 & -1.0002971485 \\
-0.0041176936 & 0.9979859780 & -1.0009837884 & -1.0004756378 & -1.0005660223 \\
-0.0085861355 & -0.0041836454 & 0.9979713164 & -1.0009608668 & -1.0012320110 \\
-0.0184060466 & -0.0089312241 & -0.0042987506 & 0.9980091457 & -1.0027097718 \\
-0.1086298824 & -0.0003050093 & -0.0000845822 & 0.0000456939 & 0.9998338096
\end{array}\right] .}
\end{aligned}
$$

Note that $\|\Delta\|_{F} \approx 0.9709$ and $\|\Theta\|_{F} \approx 0.2395$.

For $n=10$ we find instead $d^{0}(A, E) \leq 0.00320$, slightly larger than the computed bound for $d(A, E)$.

5. Conclusions and further work. We have investigated the distance of a given regular pencil to a singular one. For the closest singular pencil we have considered two cases, one where the pencil is singular due to a common left or right null vector and the general situation where such a situation need not occur. For the first case we have proved that the distance can always be determined by rank-one perturbations both in $A$ and in $E$. For the second case we expect instead that in general the perturbations have full rank.

We have presented and analyzed algorithms based on an inner-outer iteration, where ordinary differential equations are driven into a stationary state in the inner iteration and the outer iteration solves for a scalar distance parameter. The algorithms for computing the closest pencil with a common null vector can exploit the low-rank structure of the perturbations such that the computational work grows only linearly with the dimension in the case of a sparse pencil, which makes the algorithms fast also for large sparse problems.

Possible future work is related to the analysis of problems with structure. A very brief discussion of some cases of interest is given in the following: 
- Real pencils: As we have noted in Section 2.4, in the case of real pencils and real perturbations it is sufficient to replace the vector fields by their real parts, i.e., with their projection on the space of real matrices.

- Hermitian pencils: Similarly, for Hermitian pairs it is sufficient to choose the values $\mu_{i} \in \mathbb{R}$ to guarantee that the solution of (2.11) remains Hermitian (for Hermitian initial values). The same holds for equations (3.11), (4.4).

- Sparse pencils: In the case where $A$ and $E$ have a prescribed sparsity pattern which has to be inherited also by the considered perturbations, we have to replace the vector fields in the ODEs by their orthogonal projections on the sparsity pattern structure, which is simply obtained by annihilating those entries which correspond to zero entries in the sparsity patterns of $A, E$.

Acknowledgments. The authors thank Daniel Kressner for his helpful remarks.

The first author thanks the Italian M.I.U.R. and the INdAM GNCS for financial support and also the Center of Excellence DEWS.

The research of the third author was carried out in the framework of MATHEON project D-SE1, Stability analysis of power networks and power network models supported by Einstein Foundation Berlin.

\section{REFERENCES}

[1] K. E. Brenan, S. L. Campbell, and L. R. Petzold. Numerical Solution of Initial-Value Problems in Differential Algebraic Equations. SIAM Publications, Philadelphia, PA, 2nd edition, 1996.

[2] R. Byers, C. He, and V. Mehrmann. Where is the nearest non-regular pencil. Linear Algebra Appl., 285:81-105, 1998.

[3] S. L. Campbell. Linearization of DAE's along trajectories. Z. Angew. Math. Phys., 46:70-84, 1995.

[4] E. Eich-Soellner and C. Führer. Numerical Methods in Multibody Systems. B. G. Teubner Stuttgart, 1998.

[5] F. R. Gantmacher. Theory of Matrices. Chelsea, New York, 1959.

[6] G. H. Golub and C. F. Van Loan. Matrix Computations. Johns Hopkins University Press, Baltimore, 3rd edition, 1996.

[7] N. Guglielmi, D. Kressner, and C. Lubich. Low rank differential equations for hamiltonian matrix nearness problems. Numer. Math., 129:279-319, 2015.

[8] N. Guglielmi and C. Lubich. Differential equations for roaming pseudospectra: paths to extremal points and boundary tracking. SIAM J. Numer. Anal., 49:1194-1209, 2011.

[9] N. Guglielmi and C. Lubich. Low-rank dynamics for computing extremal points of real pseudospectra. SIAM J. Matrix Anal. Appl., 34:40-66, 2013.

[10] N. Guglielmi and M. Overton. Fast algorithms for the approximation of the pseudospectral abscissa and pseudospectral radius of a matrix. SIAM J. Matrix Anal. Appl., 32:1166-1192, 2011.

[11] N. Guglielmi, M. Overton, and G. Stewart. An efficient algorithm for computing the generalized null space decomposition. SIAM J. Matrix Anal. Appl., 36:38-54, 2015.

[12] E. Hairer, C. Lubich, and M. Roche. The Numerical Solution of Differential-Algebraic Systems by Runge-Kutta Methods. Springer-Verlag, Berlin, Germany, 1989.

[13] E. Hairer and G. Wanner. Solving Ordinary Differential Equations II: Stiff and DifferentialAlgebraic Problems. Springer-Verlag, Berlin, Germany, 2nd edition, 1996.

[14] N. J. Higham. Matrix nearness problems and applications. In M. Gover and S. Barnett, editors, Applications of Matrix Theory, pages 1-27. Oxford University Press, 1989.

[15] T. Kato. Perturbation Theory for Linear Operators. Springer Verlag, New York, N.Y., 1995.

[16] Daniel Kressner and Matthias Voigt. Distance problems for linear dynamical systems. In Numerical algebra, matrix theory, differential-algebraic equations and control theory, pages 559-583. Springer, Cham, 2015.

[17] P. Kunkel and V. Mehrmann. Differential-Algebraic Equations. Analysis and Numerical Solution. EMS Publishing House, Zürich, Switzerland, 2006. 
[18] R. Lamour, R. März, and C. Tischendorf. Differential-Algebraic Equations: A Projector Based Analysis. Differential-Algebraic Equations Forum. Springer Verlag, 2013.

[19] R. B. Lehoucq, D. C. Sorensen, and C. Yang. ARPACK Users' Guide: Solution of Large-Scale Eigenvalue Problems with Implicitly Restarted Arnoldi Methods. SIAM, Philadelphia, 1998.

[20] The MathWorks, Inc., Natick, MA. 2013. http://www.mathworks.de/products/simscape/.

[21] C. Mehl, V. Mehrmann, and M. Wojtylak. On the distance to singularity via low rank perturbations. Operators and Matrices, 9:733-772, 2015.

[22] C.D. Meyer and G.W. Stewart. Derivatives and perturbations of eigenvectors. SIAM J. Numer. Anal., 25:679-691, 1988.

[23] Modelica Association. Modelica standard library 3.2.1. https://www.modelica.org/.

[24] R. Riaza. Differential-algebraic systems. Analytical aspects and circuit applications. World Scientific Publishing Co. Pte. Ltd., Hackensack, NJ., 2008. 